\title{
From Hunger to Satiety: Reconfiguration of a Feeding Network by Aplysia Neuropeptide Y
}

\author{
Jian Jing, ${ }^{1}$ Ferdinand S. Vilim, ${ }^{1}$ Charles C. Horn,${ }^{2,3}$ Vera Alexeeva, ${ }^{1}$ Nathan G. Hatcher ${ }^{4}$ Kosei Sasaki, ${ }^{1}$ Irene Yashina, ${ }^{1}$ \\ Yuriy Zhurov, ${ }^{1}$ Irving Kupfermann, ${ }^{3}$ Jonathan V. Sweedler, ${ }^{4}$ and Klaudiusz R. Weiss ${ }^{1}$ \\ ${ }^{1}$ Department of Neuroscience, Mount Sinai School of Medicine, New York, New York 10029, ${ }^{2}$ Monell Chemical Senses Center, Philadelphia, Pennsylvania \\ 19104, ${ }^{3}$ Center for Neurobiology and Behavior, Columbia University, New York, New York 10032, and ${ }^{4}$ Department of Chemistry and the Beckman \\ Institute, University of Illinois, Urbana, Illinois 61801
}

\begin{abstract}
A shift in motivational state often produces behavioral change, but the underlying mechanisms are poorly understood. In the marine mollusc, Aplysia californica, feeding-induced transition from a hunger to satiation state leads to a slowdown and an eventual termination of feeding. Because the multifunctional feeding network generates both ingestion and the competing response, egestion, it is possible that the transition from a hunger to a satiety state is associated with network reconfiguration that results in production of fewer ingestive and more egestive responses. Chronic electrophysiological recordings in free-feeding Aplysia showed that as the meal progressed, food elicited fewer ingestive responses and simultaneously increased the number of egestive responses. Injections of Aplysia neuropeptide $\mathrm{Y}$ (apNPY) reduced food intake and slowed down the rate of ingestion. apNPY was localized to buccal-ganglion afferents originating in the gut-innervating esophageal nerve (EN), a nerve involved both in satiation and in the generation of egestive programs. During EN stimulation, apNPY was released in the feeding circuit. Importantly, stimulation of the cerebral-buccal interneuron-2, a command-like interneuron that is activated by food and normally elicits ingestive responses, elicited egestive responses in the presence of apNPY. This was accompanied by increased activity of the egestion-promoting interneuron B20 and decreased activity in the ingestion-promoting interneuron B40. Thus, apNPYergic reconfiguration of the feeding central pattern generator plays a role in the gradual transition from hunger to satiety states. More generally, changes in the motivational states may involve not only simple network inhibition but may also require network reconfiguration.
\end{abstract}

Key words: PYY; feeding; satiation; ingestion; egestion; motivational state

\section{Introduction}

An organism's motivational state is a major determinant of the expression of various behaviors. Most commonly, the motivational state is altered through consummatory activity. For instance, feeding behaviors are organized into a behavioral sequence in which food ingestion leads to termination of feeding. This behavioral transition is effected by a shift in the internal state (i.e., a progressive decrease of hunger and establishment of a satiety state). A common thread emerging from vertebrate and invertebrate work suggests that termination of feeding may be determined by a balance of the direct excitatory actions of orosensory stimuli and inhibitory actions of postingestive stimuli that converge on the feeding central pattern generator (CPG) that mediates ingestive behaviors (Kupfermann, 1974; Susswein and Kupfermann, 1975; Davis et al., 1977; Reingold and Gelperin, 1980; Groome et al., 1993; Smith, 2000; Horn et al., 2001; Grill,

Received Jan. 24, 2007; revised Feb. 21, 2007; accepted Feb. 23, 2007.

This work was supported by National Institutes of Health Awards R01 MH 50235, MH 035564, DA 13330, and R01 NS 031609 (J.V.S.) and by National Institute on Drug Abuse Award P30 DA 018310 to the University of Illinois at Urbana-Champaign Neuroproteomics Center.

Correspondence should be addressed to Dr. Jian Jing, Department of Neuroscience, Mount Sinai School of Medicine, Box 1065, 1 Gustave Levy Place, New York, NY 10029. E-mail: Jian.Jing@mssm.edu.

D01:10.1523/JNEUROSCI.0334-07.2007

Copyright $\odot 2007$ Society for Neuroscience $\quad$ 0270-6474/07/273490-13\$15.00/0
2006). Furthermore, several "satiety" peptides have been implicated in the inhibitory process leading to the development of satiety states (Schwartz et al., 2000; Smith, 2000; Badman and Flier, 2005; Cone, 2005; Morton et al., 2006). However, because the transition from hunger to satiety states is a gradual and dynamic process, it is unlikely that this process is mediated via a fast switch mechanism (e.g., the transitions between sleep-wake states) (Lu et al., 2006). Moreover, it is presently not clear how peptidergic inhibition of a CPG may be implemented at the neuronal level to bring about satiety.

An interesting possibility for an alternative, but not exclusive, mechanism stems from the fact that the feeding CPG is a multifunctional neural network that generates competing ingestive and egestive responses through recruitment of specific CPG elements. This multifunctional organization is present in both invertebrates (Combes et al., 1999; Jing and Weiss, 2001, 2002) and vertebrates (DiNardo and Travers, 1997; Altschuler, 2001; Hornby, 2001; Chen and Travers, 2003). Notably, multifunctional networks are subject to reconfiguration by neuromodulators (Harris-Warrick and Marder, 1991; Lieske et al., 2000; Kupfermann and Weiss, 2001; Nusbaum and Beenhakker, 2002; Kristan et al., 2005; Grillner, 2006), such as peptides. Thus, it is plausible that reconfiguration of the feeding CPG (e.g., selective inhibition of ingestion-promoting elements and recruitment of 
egestion-promoting elements) terminates feeding. Yet, such a possibility has not been examined previously.

Here, we exploited advantageous features of the Aplysia californica feeding network to determine whether network reconfiguration is involved in the transition from the hunger to satiation state. We used chronic electrophysiological recordings in free-feeding animals to study various food-evoked responses while the animals were fed to satiation. We examined the role of Aplysia neuropeptide Y (apNPY) (Rajpara et al., 1992) in satiation by determining its localization, release, and action on food intake and the feeding circuit, because it is a homolog of the vertebrate pancreatic polypeptide (PP) family that includes NPY and peptide YY (PYY), that play a role in vertebrate feeding (e.g., Batterham et al., 2002; Kalra and Kalra, 2004). Our study suggests that the gradual transition from hunger to satiation may be mediated in part by the apNPYergic reconfiguration of the feeding CPG.

\section{Materials and Methods}

Aplysia californica were obtained from Marinus Scientific (Garden Grove, CA) and maintained in circulating artificial seawater (ASW) at $14-15^{\circ} \mathrm{C}$.

Behavioral experiments. Aplysia (100-200 g) were food-deprived for $4-7 \mathrm{~d}$ before experiments. Motor programs were monitored by buccal nerve recordings from intact animals that were fed to satiation. Electrodes for en passant recordings were implanted as described previously (Morton and Chiel, 1993a; Horn and Kupfermann, 2002). Animals that recovered fully from surgery were fed continuously with $1 \times 10 \mathrm{~cm}$ seaweed strips until they stopped ingesting seaweed for $3 \mathrm{~min}$. Data were digitized using Digidata acquisition system and Axoscope software (Molecular Devices, Sunnyvale, CA).

Motor programs were classified using fractional overlaps between bursts of activity in the radula nerve (RN) and the buccal nerve 2 (BN2) as defined previously (Morton and Chiel, 1993a). Specifically, for each motor program, we characterized the duration of the burst of large units in the RN (see Fig. $1 A$, gray bars). Large units were detected using the "threshold search" of Clampfit software (Molecular Devices). The onset of the burst in BN2 (see Fig. $1 A$, arrows) was identified as near or after the onset of RN burst and after a characteristic brief inactivity in BN2. Fractional overlap was then calculated as the duration of the RN burst that overlaps with the BN2 burst (see Fig. $1 A$, blue bars) divided by the duration of total RN burst. Previously (Morton and Chiel, 1993a), it was shown that BN2 bursts represent a retraction phase, and larger units of $\mathrm{RN}$ bursts originate from the radula closure motoneuron $\mathrm{B} 8$. Thus, a high fractional overlap $(0.8-1)$ is associated with ingestive responses, because the radula is closed during retraction to take in food. A low fractional overlap $(0-0.1)$ is associated with egestive responses, because the radula is closed during protraction, which precedes retraction, to push out food. Intermediate values of fractional overlap (0.1-0.8) were classified as intermediate responses, because they did not unequivocally define either ingestion or egestion.

In a separate series of experiments, effects of apNPY on feeding were examined. In each experiment, a pair of animals was housed in separate round net cages ( $17 \mathrm{~cm}$ diameter and $17 \mathrm{~cm}$ height) that were placed in a $500 \mathrm{~L}$ tank filled with circulating aerated ASW $\left(14-16^{\circ} \mathrm{C}\right)$. To assure that the animals were responsive to food, they were first pretested with a small piece of seaweed. After this pretest, in each pair, one animal was injected (into the hemocoel using a syringe needle) with apNPY freshly dissolved in $1.5 \mathrm{ml} \mathrm{ASW}$, whereas the other was injected with $1.5 \mathrm{ml}$ of ASW. Three minutes later, the feeding session began. Animals were fed seaweed (Shirako, Tokyo, Japan) cut in $1 \times 10 \mathrm{~cm}$ strips. Each strip of seaweed was delivered to the mouth area with forceps. Feeding was terminated when animals stopped ingesting seaweed for $>3 \mathrm{~min}$. The experimenter who fed the animals did not know which animal was injected with apNPY.

In situ hybridization. In situ hybridization was performed as described previously (Vilim et al., 2001). Briefly, after digestion with protease IX to facilitate the removal of sheath, the ganglia were washed and fixed overnight with $4 \%$ paraformaldehyde in PBS. After a wash with PBS and $0.1 \%$ Tween 20 (PBT), the ganglia were then desheathed and dehydrated in an ascending methanol series and stored in $100 \%$ methanol overnight at $-20^{\circ} \mathrm{C}$. The ganglia were then rehydrated and washed with PBS and $0.3 \%$ Triton X-100 and refixed for 20 min with 4\% paraformaldehyde in PBS. After washing with PBS, the reactive groups were neutralized by washing first with $2 \mathrm{mg} / \mathrm{ml}$ glycine in PBS, then with $0.1 \mathrm{M}$ triethanolamine- $\mathrm{HCl}$ (TEA-HCl), $\mathrm{pH} 8.0$, and finally with $1 \mathrm{ml}$ of TEA-HCl containing $2.5 \mu \mathrm{l}$ of acetic anhydride. After washing with PBT, the ganglia were prehybridized in hybridization buffer (Hyb-buffer) (50\% formamide, 5 mM EDTA, $5 \times$ SSC, $1 \times$ Dernhardt's solution, $0.1 \%$ Tween 20 , and $0.5 \mathrm{mg} / \mathrm{ml}$ yeast tRNA) overnight at room temperature and for $6-8 \mathrm{~h}$ at $50^{\circ} \mathrm{C}$ with fresh Hyb-buffer.

Digoxigenin-labeled cRNA was synthesized with T7 RNA polymerase using the apNPY cDNA clone as a template and used as a probe at 2 $\mu \mathrm{g} / \mathrm{ml}$ in Hyb-buffer. The ganglia were hybridized overnight at $50^{\circ} \mathrm{C}$ and washed at $50^{\circ} \mathrm{C}(30 \mathrm{~min}$ each in $50 \%$ formamide, $5 \times$ SSC, and $1 \%$ SDS; in $50 \%$ formamide, $2 \times$ SSC, and $1 \%$ SDS; in $50 \%$ formamide, $2 \times$ SSC, and $1 \%$ SDS; and in $0.2 \times$ SSC). After more washes with PBT containing $0.2 \%$ BSA and blocked for $2 \mathrm{~h}$ with PBT containing $0.2 \%$ BSA and $10 \%$ normal goat serum (NGS), the ganglia were incubated overnight at $4^{\circ} \mathrm{C}$ with a 1:200 dilution of alkaline phosphatase-conjugated antidigoxigenin antibody in PBT containing $0.2 \%$ BSA and $1 \%$ NGS. After washes with PBT, the ganglia were washed with detection buffer $(100 \mathrm{~mm} \mathrm{NaCl}$, $50 \mathrm{~mm} \mathrm{MgCl}_{2}, 0.1 \%$ Tween 20, $1 \mathrm{~mm}$ levamisol, and $100 \mathrm{~mm}$ TrisHCl, pH 9.5) and developed with $4.5 \mu \mathrm{l}$ of nitroblue tetrazolium and $3.5 \mu \mathrm{l}$ of 5-bromo-4-chloro-3-indolyl phosphate in $1 \mathrm{ml}$ of detection buffer (Roche, Indianapolis, IN). The staining reaction was monitored visually and stopped by washing with PBT. The ganglia were photographed using a Nikon microscope (Morrell Instruments, Melville, NY) with epiillumination against a white background.

Antibodies. Because the C-terminal apNPY contains RFamide motif that is present in a number of other peptides in Aplysia (Cropper et al., 1994), we opted to generate an antibody in rats against a 18 amino-acid synthetic peptide (SynPep, Dublin, CA) at the apNPY N terminal as described previously (Furukawa et al., 2001; Sweedler et al., 2002). Briefly, the antigen was prepared by coupling synthetic peptide to BSA (Sigma-Aldrich, St. Louis, MO) using 1-ethyl-3-(dimethylaminopropyl)carbodiimide (EDC; Sigma-Aldrich). The coupling was performed and allowed to react overnight at $4^{\circ} \mathrm{C}$ in $1 \mathrm{ml}$ of $50 \mathrm{~mm} \mathrm{NaH}_{2} \mathrm{PO}_{4}$, $\mathrm{pH} 7.2$, containing $10 \mathrm{mg}$ of BSA, $1 \mathrm{mg}$ of peptide, and $25 \mathrm{mg}$ of EDC. The coupled antigen was purified from the reaction using a Microcon-30 spinning at $13,800 \times \mathrm{g}$ for $30 \mathrm{~min}$ at $4^{\circ} \mathrm{C}$. After washing the retentate four times with $0.4 \mathrm{ml}$ of $50 \mathrm{~mm} \mathrm{NaH}_{2} \mathrm{PO}_{4}$, it was resuspended in $0.5 \mathrm{ml}$ of the same buffer and transferred to a new tube.

Two male Sprague Dawley rats (250-300 g; Taconic, Germantown, NY) were immunized by intraperitoneal injection with either $12.5 \mu \mathrm{l}$ ( $250 \mu \mathrm{g}$; rat 1$)$ or $25 \mu \mathrm{l}(500 \mu \mathrm{g}$; rat 2$)$ of antigen in an emulsion of $0.3 \mathrm{ml}$ of PBS and $0.3 \mathrm{ml}$ of Freund's complete adjuvant. At days 21 and 42, the rats were boosted by intraperitoneal injection with either $6.25 \mu \mathrm{l}(125 \mu \mathrm{g}$; rat 1$)$ or $12.5 \mu \mathrm{l}(250 \mu \mathrm{g}$; rat 2$)$ of antigen in the emulsion as above. The animals were killed by decapitation at $49 \mathrm{~d}$, and the blood was harvested and processed for serum. Sera were aliquoted, frozen, and lyophilized, or stored at $4^{\circ} \mathrm{C}$ with EDTA ( $25 \mathrm{~mm}$ final concentration) and thimerosal ( $0.1 \%$ final concentration) added as stabilizers. Specificity of immunostaining was confirmed by preincubation of the primary antibody with $10^{-4} \mathrm{M}$ of the synthetic apNPY, which abolished the staining.

Immunocytochemistry. Immunocytochemistry was performed as described previously (Furukawa et al., 2001). Tissues were fixed in $4 \%$ paraformaldehyde, $0.2 \%$ picric acid, $25 \%$ sucrose, and $0.1 \mathrm{M} \mathrm{NaH}_{2} \mathrm{PO}_{4}$, $\mathrm{pH} 7.6$, for either $3 \mathrm{~h}$ at room temperature or overnight at $4^{\circ} \mathrm{C}$. After washes with PBS, ganglia were desheathed to expose the neurons except in ganglia from small animals $(10-15 \mathrm{~g})$. All subsequent incubations were done at room temperature. Tissue was permeabilized and blocked by overnight incubation in blocking buffer (BB) $(10 \%$ normal donkey serum, 2\% Triton X-100, 1\% BSA, $154 \mathrm{~mm} \mathrm{NaCl}, 50$ mм EDTA, $0.01 \%$ thimerosal, and $10 \mathrm{~mm} \mathrm{Na}_{2} \mathrm{HPO}_{4}, \mathrm{pH}$ 7.4). The primary antibody was diluted 1:250 in $\mathrm{BB}$ and incubated with the tissue for 4-7 $\mathrm{d}$. The tissue 
was then washed twice per day for 2-3 d with washing buffer (WB) (2\% Triton X-100, 1\% BSA, $154 \mathrm{~mm} \mathrm{NaCl}, 50$ mm EDTA, 0.01\% thimerosal, and $10 \mathrm{~mm} \mathrm{Na}_{2} \mathrm{HPO}_{4}, \mathrm{pH}$ 7.4). The tissue was then incubated with a 1:500 dilution of secondary antibody (lissamine rhodamine donkey antirat; Jackson ImmunoResearch, West Grove, PA) for 2-3 d. The tissue was then washed twice with WB for $1 \mathrm{~d}$ and four times with storage buffer ( $1 \%$ BSA, $154 \mathrm{~mm} \mathrm{NaCl}, 50 \mathrm{~mm}$ EDTA, 0.01\% thimerosal, and $10 \mathrm{~mm}$ $\mathrm{Na}_{2} \mathrm{HPO}_{4}$, $\mathrm{pH} 7.4$ ) for $1 \mathrm{~d}$.

For double-labeling experiments, neurons were identified based on morphology and electrophysiological characters and injected with carboxyfluorescein (Jing et al., 2003), and the tissue was fixed and processed for immunocytochemistry as described above. In nerve ligation experiments, a small cut was made in $\mathrm{MgCl}_{2}$-anesthetized animals at the side of the body between rhinophore and foot. The esophageal nerve (EN) was exposed and tied. The animals were sutured and returned to the tank for $1 \mathrm{~d}$. These animals were then killed, and the tissues were dissected, fixed, and processed for immunocytochemistry.

Electrophysiology. Conventional electrophysiological recordings were performed as described previously (Jing and Weiss, 2005). The cerebral and buccal ganglia were desheathed. The preparation was then transferred to a recording chamber, lined with sylgard containing $\sim 1.5 \mathrm{ml}$ of ASW (in mM: $460 \mathrm{NaCl}, 10 \mathrm{KCl}, 55 \mathrm{MgCl}_{2}, 11 \mathrm{CaCl}_{2}$, and $10 \mathrm{HEPES}$ buffer, $\mathrm{pH}$ 7.6), continuously perfused with ASW at $0.3 \mathrm{ml} / \mathrm{min}$, and maintained at $14-17^{\circ} \mathrm{C}$. Peptides were dissolved in ASW immediately before each application. Nerve stimulation and recording were done with polyethylene suction electrodes. The egestive motor programs were evoked by stimulation of the posterior branch of the EN (supplemental Fig. 1, available at www.jneurosci.org as supplemental material) with $8-15 \mathrm{~V}$ and $3 \mathrm{~ms}$ pulses at $2 \mathrm{~Hz}$ and monitored with intracellular recording of the radula closer motoneuron (B8) and other output neurons.

Matrix-assisted laser desorption/ionization time-of-flight mass spectrometry. Matrix-assisted laser desorption/ionization time-of-flight (MALDI TOF) mass spectrometry (MS) was performed with either a Voyager-DE-STR mass spectrometer (Applied Biosystems, Foster City, CA) or an Ultraflex II MALDI TOF mass spectrometer (Bruker Daltonics, Billerica, MA). Releasate mass spectra were obtained in linear mode with each spectrum averaging 300-400 laser pulses. Mass spectra were initially calibrated externally with peptide standards and in some cases recalibrated with spiked internal calibrants (Hatcher et al., 2005).

Tissues from the EN and the rostral buccal regions immunopositive for apNPY were manually dissected, rinsed in $\mathrm{H}_{2} \mathrm{O}$, and transferred to a MALDI target plate (either ABI gold-plated targets or Bruker Daltonics PAC 384 prespotted targets) for MS analyses. Extraction was performed on the target surface with $\sim 0.5 \mu \mathrm{l}$ of acidified acetone (40 acteone:6 $\mathrm{H}_{2} \mathrm{O}: 1 \mathrm{HCl}, 12 \mathrm{~N}$ ). CHCA ( $\alpha$-cyano-4-hydroxycinnamic acid) MALDI matrix was applied to the sample spot after extraction. CHCA MALDI matrix was mixed in $70 \%$ acetonitrile $(\mathrm{ACN})$ with $0.01 \%$ trifluoro acetic acid. Reagents were obtained from Sigma-Aldrich.

Solid-phase extraction bead sampling of peptidergic releasates. The solidphase extraction beads were Resin "D" (International Sorbent Technology, Hengoed, UK) that were "wetted" in $50 \%$ ACN and equilibrated in ASW. Individual, activated beads were transferred between solutions or Aplysia ganglions by hand-held borosilicate capillary pipettes (World Precision Instruments, Sarasota, FL) with tips pulled to $\sim 200 \mu \mathrm{m}$ in diameter. Beads were positioned on the rostral surface of isolated buccal ganglia (supplemental Fig. 1, available at www.jneurosci.org as supplemental material), where apNPY-immunopositive processes are abundant (see Fig. 3B1). To limit dilution of releasates, perfusion was suspended in this set of experiments. To discriminate activity-dependent secretion of neuropeptides from background compounds, bead samples were collected for 10 min intervals before and during EN-elicited egestive motor programs; new beads were repositioned in the same location for each sampling period. After collection, beads were transferred from the ganglia, washed of salts in Millipore (Bedford, $\mathrm{MA}$ ) $\mathrm{H}_{2} \mathrm{O}$, and transferred to MALDI targets. Analytes were eluted from the beads onto the MALDI target surface by rinsing the beads first with $70 \% \mathrm{ACN} / \mathrm{H}_{2} \mathrm{O}(\sim 0.2 \mu \mathrm{l})$ and then with $70 \% \mathrm{ACN} / \mathrm{H}_{2} \mathrm{O}$ containing saturated CHCA MALDI ma$\operatorname{trix}(\sim 0.2 \mu \mathrm{l})$.

Data analysis. Digitized electrophysiological recordings were plotted using Coreldraw (Ottawa, Ontario, Canada) and Axum (Mathsoft, Cambridge, MA). Data are expressed as mean \pm SEM. Differences between two data groups were tested with a two-tailed paired or unpaired $t$ test. Data with more than two groups were first analyzed with one-way ANOVA to show overall significant difference among these groups. Individual comparisons between groups were subsequently made with Bonferroni corrections. All statistical tests were performed using GraphPad Prism 4.0 (GraphPad, San Diego, CA).

\section{Results}

\section{Dynamics of motor program generation during a meal}

As a meal progressed, we observed that animals took longer to consume a strip of seaweed as the animals' feeding became less effective. In some cases, small alternating in and out movements from the buccal cavity were observed. However, the nature of some of these food-evoked responses was not unequivocally determined from visual observation, because the feeding apparatus (radula) is partially internal and therefore some responses may be covert (Kupfermann, 1974; Morton and Chiel, 1993a). Thus, we performed in vivo nerve recordings $(n=3)$ to determine whether the ineffective feeding during the later portion of a meal was caused by a reduction of the fraction of ingestive responses and/or to an increase of the fraction of egestive responses. We took advantage of a previous finding reporting that in freely feeding animals, ingestive and egestive responses were distinguished on the basis of recordings from two buccal nerves: buccal nerve 2 and the radula nerve (Morton and Chiel, 1993a).

To evaluate the potential changes of behavioral responses during a meal, we divided the entire meal into three periods: (1) the "start" period, consisting of the first one-third amount of seaweed consumed; (2) the "middle" period, where the second onethird amount of seaweed was consumed; and (3) the "end" period, where the last one-third amount of seaweed was eaten. Figure $1 A$ illustrates a series of motor programs recorded in the start (top) and the end (bottom) periods. As described in Materials and Methods, we determined the fractional overlap of the $\mathrm{RN}$ and BN2 bursts and classified each program during the meal as "ingestive," "egestive," or "intermediate" responses and calculated the percentage of the three subtypes of programs in each of the three parts of the meal (start, middle, and end). The data (Fig. $1 B$ ) showed that as the meal progressed, the fraction of ingestive responses $\left(F_{(2,4)}=146.1 ; p<0.001\right)$ decreased, whereas the fraction of intermediate $\left(F_{(2,4)}=63.3 ; p<0.001\right)$ and egestive responses $\left(F_{(2,4)}=109.1 ; p<0.001\right)$ increased. During the onset of satiation, a lower efficiency of feeding was observed before eventual termination. These events were associated not only with a reduction of the fraction of ingestive responses, but also an elevated fraction of responses that actively prevented ingestion of food (i.e., egestive responses).

\section{Inhibition of food intake by apNPY}

In apNPY experiments, animals were injected with either ASW $(n=11), 0.47 \mathrm{mg} / \mathrm{kg}$ apNPY $(n=6)$, or $4.7 \mathrm{mg} / \mathrm{kg}$ apNPY $(n=$ $5)$. apNPY doses corresponded approximately to final hemocoel concentrations between $10^{-7} \mathrm{M}$ and $10^{-6} \mathrm{M}$. Animals consumed $17.9 \pm 1.0$ (mean \pm SEM) strips of seaweed after ASW injection, $4.8 \pm 0.9$ strips of seaweed after $0.47 \mathrm{mg} / \mathrm{kg}$ apNPY injection, and $0.9 \pm 0.3$ strips of seaweed after injection of $4.7 \mathrm{mg} / \mathrm{kg}$ apNPY. Each strip of dried seaweed weighed $\sim 0.1 \mathrm{~g}$ and weighed 10 times greater when hydrated. The amount of seaweed consumed was converted to hydrated weight and normalized to animal body weight (average body weight, $0.138 \mathrm{~kg}$ ) (Fig. 1C1). Normalized food intake differed significantly between groups $\left(F_{(2,19)}=56.2\right.$; $p<0.0001$, one-way ANOVA). Injections of apNPY reduced 

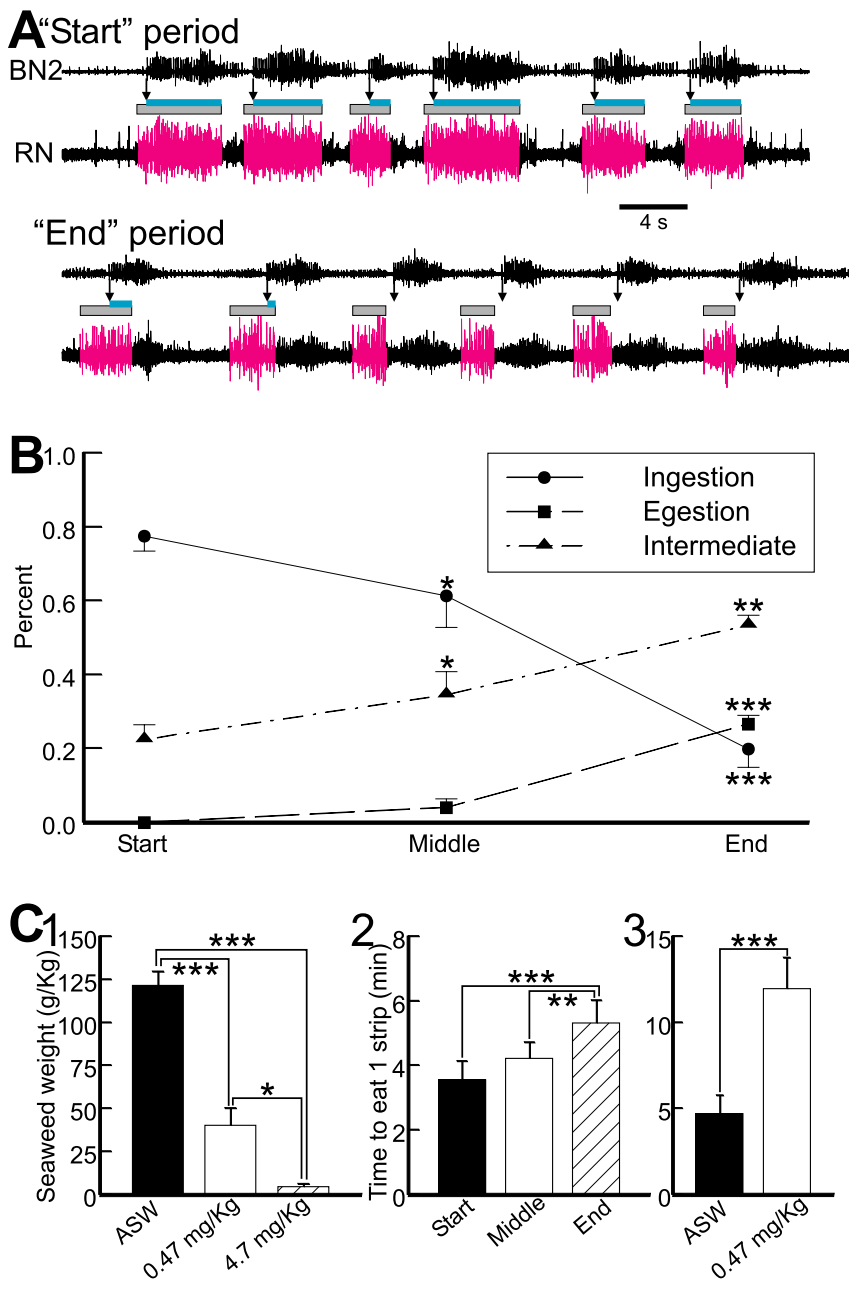

Figure 1. Behavioral and physiological actions of satiation and apNPY. Each meal is divided into three periods based on the amount of seaweed consumed: the start (first one-third of seaweed), the middle (second one-third of seaweed), and the end (last one-third of seaweed) periods. $\boldsymbol{A}$, Representative examples of motor programs recorded during the start period versus the end period of a meal. Each cycle of motor program is characterized by an RN burst of larger units (red, gray bars). The onset of BN2 bursts (arrows) relative to the RN burst determines the fractional overlap (blue lines over gray bars) between RN and BN2 bursts. In the start period, the programs were mostly ingestive, because the BN2 bursts essentially overlapped with the RN bursts. In the end period, the programs were mostly egestive, because the RN bursts did not overlap with BN2 bursts, except that the first program was intermediate as the RN burst partially overlapped with the BN2 burst. $\boldsymbol{B}$, Percentage of ingestive, egestive, and intermediate responses during the start, middle, and end portions of a meal $(n=3)$. C1, apNPY reduced the amount of food (seaweed) consumed in a single meal in a dose-dependent manner. $\mathbf{C}$, As the meal progressed from the start, through the middle, to the end periods, control animals took longer to consume single strips of seaweed $(1 \times 10 \mathrm{~cm})$. C3, Animals injected with $0.47 \mathrm{mg} / \mathrm{kg}$ apNPY took longer to consume one strip of seaweed (average of the first three strips) compared with control animals injected with ASW. Post hoc tests with Bonferroni corrections in $\boldsymbol{B}$ and $\boldsymbol{C}$ : ${ }^{*} p<0.05 ;{ }^{* *} p<0.01 ;{ }^{* * *} p<0.001$. Stars in B indicate significant difference compared with start periods. The error bar represents SEM.

food intake in a dose-dependent manner. Because animals in the $4.7 \mathrm{mg} / \mathrm{kg}$ apNPY group ate a negligible amount of seaweed, these animals were retested the next day to determine whether they had recovered from apNPY treatment. These animals consumed $96.7 \pm 5.6 \mathrm{~g} / \mathrm{kg}$, which is not significantly different from the amount consumed by the ASW-injected animals on the preceding day $(112.7 \pm 11.8 \mathrm{~g} / \mathrm{kg} ; p>0.05$, two-tailed $t$ test $)$. Thus, the effects of apNPY on the suppression of feeding did not extend to the second day after injection.
Similar to the experiments in the last section, we observed that as a meal progressed and the animals approached satiation, the animals tended to take longer to consume individual strips of seaweed. Specifically, we found that the animals took longer to ingest a single strip of seaweed during the middle and end periods of the meal than they did during the start period (Fig. 1C2) $\left(F_{(2,20)}=18.11 ; p<0.0001\right)$. To determine whether apNPYinjected animals already behaved at the beginning of the meal in a manner similar to control animals that were approaching satiation, we calculated the average time to consume a single strip based on the consumption of the first three strips in the meal. Animals injected with apNPY took a longer time to eat one strip of seaweed than control animals (Fig. 1C3) $(p<0.01$; two-tailed $t$ test).

Localization and distribution of apNPY-positive neurons and processes in the feeding system

We performed whole-mount in situ hybridization and immunocytochemistry, and localized apNPY in several buccal and cerebral somata within the Aplysia feeding system (Figs. 2, 3) (for a schematic diagram of the CNS and neuronal elements that are involved in feeding, see supplemental Fig. 1, available at www. jneurosci.org as supplemental material). Comparison of the distribution of neuronal somata staining for apNPY with in situ hybridization and immunocytochemistry revealed a remarkable similarity. In both in situ hybridization and immunocytochemistry of the cerebral ganglion (Fig. 2), the majority of apNPY staining was on the dorsal surface with few apNPY-positive neurons on the ventral surface. Notably, on the dorsal surface, there was a characteristic neuron (Fig. 2, arrowheads) between the roots of anterior tentacular (AT) and upper labial (UL) nerves. In the middle of the ganglion near the $\mathrm{C}$ and $\mathrm{D}$ clusters, there was one medium- to large-sized neuron together with several smaller, adjacent neurons. Importantly, there appeared to be no apNPYpositive neurons that corresponded to the $\mathrm{M}$ or $\mathrm{E}$ cluster cerebral-buccal interneurons (CBIs) on the ventral surface of the cerebral ganglion. CBIs are initiators or modulators of feeding motor programs (Rosen et al., 1991; Xin et al., 1999; Morgan et al., 2002; Hurwitz et al., 2003; Jing et al., 2004).

In the buccal ganglion, both in situ hybridization and immunostaining revealed a single strongly stained apNPY-positive neuron on the rostral surface (Fig. $3 A, B$ ). Backfill of CBCs combined with immunocytochemistry indicated that this neuron is a buccal-cerebral interneuron (BCI) that sends its sole axon through the ipsilateral $\mathrm{CBC}$ to the cerebral ganglion (Fig. 3C1,C2). This BCI neuron was identified as B18 (Chiel et al., 1988) based on its physiological properties (Figs. 4, 7), morphology via injected carboxylfluorescein (Fig. 3D1), and immunocytochemistry methods (Fig. 3D2).

Using the apNPY antibody, we visualized apNPY-positive fibers/processes. Strikingly, the staining showed that although there were relatively few apNPY-positive somata, there were many apNPY-positive fibers in the neuropile, as well as the nerves and connectives of the cerebral and buccal ganglia. In the cerebral ganglion (Fig. 2 B), apNPY-positive fibers were present in all major connectives (cerebral-buccal, cerebral-pedal, and cerebralpleural), whereas few were present in the AT, UL, and posterior tentacular nerves that innervate the head, suggesting that little apNPY originates from head sensory organs. In the buccal ganglion (Fig. $3 B$ ), the four buccal nerves (nerves 1, 2, 3, and radula nerve) that innervate the buccal mass were devoid of staining. Prominent staining was present in the EN as well as the neuropile, particularly in the area where the EN enters the ganglion. Staining 
in the EN was almost exclusively located in the posterior branch, which innervates the esophagus and the gut, but was mostly absent in the anterior branch, which primarily innervates the buccal mass (Schwarz and Susswein, 1986) (Fig. 3F).

Nerve ligation experiments were used to determine the origin of the apNPYpositive fibers in the EN $(n=4)$. Because peptides are synthesized in cell bodies and transported anterogradely (Strand, 1999), this procedure leads to peptide accumulation at the ligature site proximal to the cell bodies. Figure $3 E$ shows that the nerve site proximal to the buccal ganglia had minimal staining, whereas the site distal from the ganglia showed strong staining. These data indicate that most apNPY fibers in the EN originate outside the buccal ganglia. Thus, apNPY fibers likely provide an apNPYergic input to the buccal ganglia.

\section{MS detection of buccal apNPY}

Additional evidence for the presence of apNPY in the feeding system was obtained using MALDI TOF MS of extracts of the part of the EN that is proximal to the buccal ganglion and of the rostral neuropilar area adjacent to the EN, both of which immunostain for apNPY. apNPY was detected in mass spectra from these extracts (Fig. 5A). In addition, two additional peptides from the apNPY prohormone were detected; the apNPYderived C-terminal peptide was observed in an unmodified (CPON) and pyro-glutamate posttranslationally modified forms (pGluCPON), as has been reported previously (Romanova et al., 2006). These data corroborate apNPY immunostaining of the EN and buccal neuropile. Furthermore, several neuropeptides that have been identified previously were detected, most notably small cardioactive peptide $\mathrm{A}\left(\mathrm{SCP}_{\mathrm{A}}\right), \mathrm{SCP}_{\mathrm{B}}, \mathrm{FRF}_{\mathrm{A}}, \mathrm{FRF}_{\mathrm{C}}$, and $\mathrm{FMRFamide}$.

\section{Release of apNPY within the buccal ganglia during stimulation of the EN}

The posterior branch of the EN is known to contain afferent fibers from the esophagus to the buccal ganglion. Electrical stimulation of this branch of EN can activate egestive motor programs (Chiel et al., 1988; Morgan et al., 2002; Zhurov et al., 2005). To determine the potential role of apNPY in the generation of egestive programs elicited by stimulation of EN afferents, we examined the complement of neuropeptides secreted after stimulation of the egestive motor programs using solid-phase extraction bead probes. The beads ( $\sim 30 \mu \mathrm{m}$ in diameter) were placed on the rostral side of the buccal ganglion near the neuropilar area where immunocytochemistry showed prominent staining (for approximate locations of bead placement, see supplemental Fig. 1, available at www.jneurosci.org as supplemental material) and replaced between prestimulation and stimulation collection periods ( $10 \mathrm{~min} ; n=3$ ). Figure $5 B$ compares mass spectra of bead samples collected before versus during EN stimulation. apNPY and pGluCPON were readily observed in the stimulation sample obtained from beads located on the buccal ganglion region ipsilateral to EN stimulation. In contrast, mass spectra of the samples obtained from either the prestimulation samples or from beads collected after stimulation on the side contralateral to the stimulated EN showed no apNPY or pGluCPON neuropeptides. Thus, these data provide compelling evidence that apNPY is present in the EN and is released at the site of the buccal CPG after stimulation of the EN.

\section{B63 interneurons, but not apNPY, play a role in initiating programs}

To summarize, we discovered two major sources of apNPY in the Aplysia feeding system: the BCI B18 and the EN. As stated above, the EN is an important afferent pathway for activation of egestive programs (Chiel et al., 1988; Morgan et al., 2002; Zhurov et al., 2005) that function to reject food, and we have shown that during a satiating meal, intermediate and egestive responses became more frequent. To elucidate the neural mechanism of apNPY actions, we used isolated CNS preparations where motor program generation can be monitored by the activity of important motor neurons and CPG interneurons of the feeding circuitry. Each cycle of motor programs consists of alternating radula protraction-retraction phases (Figs. 6,7A), whereas the activity of radula closure motoneuron B8 is variably coupled to protraction or retraction. Motor programs are classified as ingestive when B8 is predominantly active during retraction, egestive when B8 is predominantly active during protraction, and intermediate when B8 is active during both protraction and retraction (Morton and Chiel, 1993a,b; Morgan et al., 2002).

Importantly, Aplysia feeding behaviors are stimulus dependent in that animals will not initiate feeding behaviors without an external stimulus. Similarly, in the isolated buccal ganglion, the CPG network is quiescent in the absence of external inputs. Two major sources of inputs are the cerebral-buccal interneuron-2 (CBI-2) and the EN (supplemental Figs. 1, 2, available at www. 

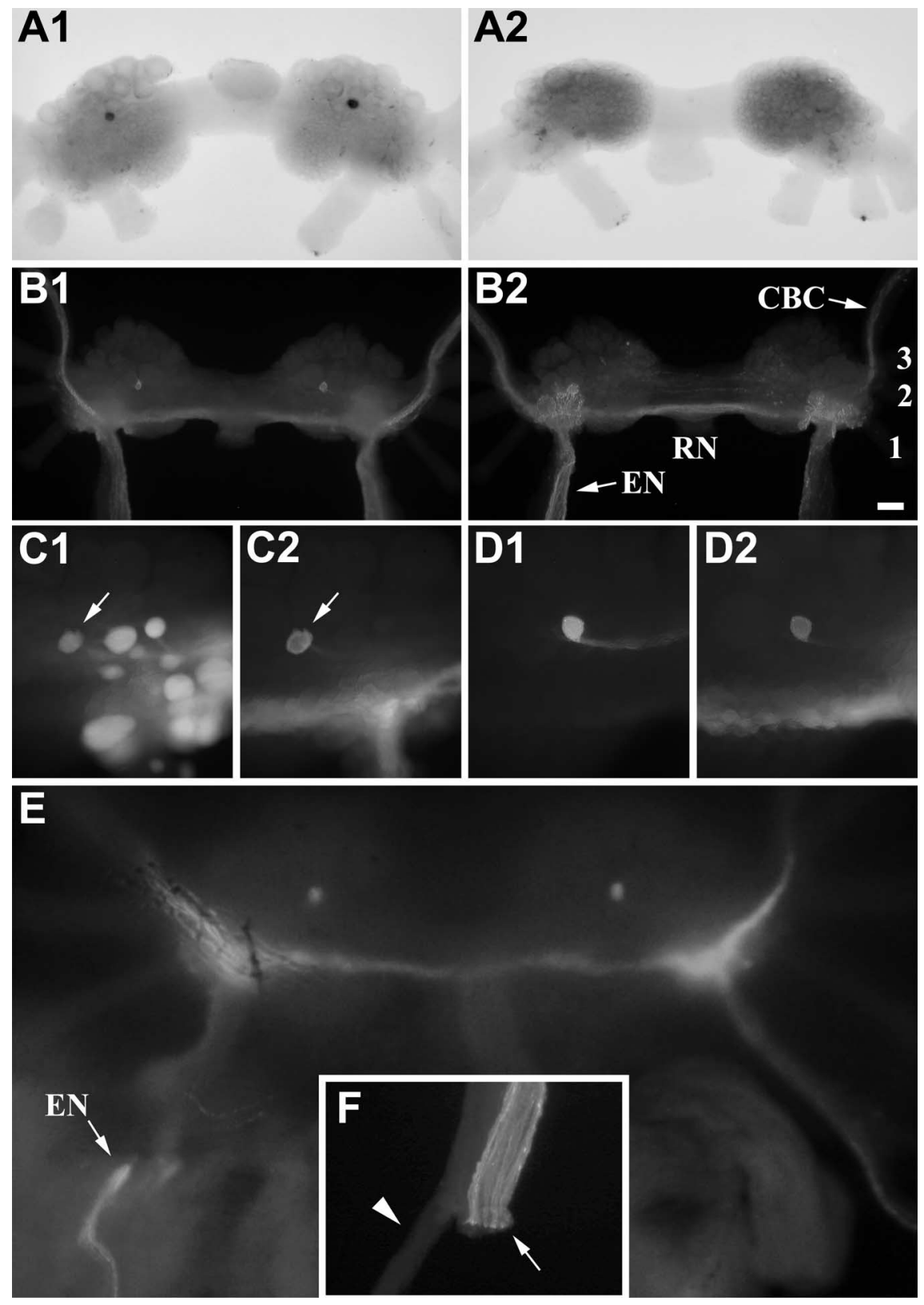

Figure 3. Localization of apNPY-positive neurons and processes in the buccal ganglion. $\boldsymbol{A}$, In situ hybridization showing the rostral (A1) and caudal (A2) surface of a buccal ganglion. $\boldsymbol{B}$, Immunocytochemistry showing the rostral (B1) and caudal (B2) surface of a different ganglion. Note the similar pattern of somata staining using either in situ hybridization $(\boldsymbol{A})$ or immunocytochemistry ( $\boldsymbol{B}$ ). Immunocytochemistry also showed apNPY-positive fibers in the CBCs and the ENs. C, Backfill of CBC with biocytin (C1) showed that one of the buccal-cerebral interneurons ( $\mathbf{C}$, arrow) is apNPY immunopositive ( $\mathbf{C}$, arrow). $\boldsymbol{D}, \mathbf{B} 18$, which was injected with dye (carboxyfluorescein; D1), is apNPY immunoreactive (D2), indicating that the buccal-cerebral interneuron in C is B18. $E$, Immunocytochemistry showing rostral view of a preparation where the left EN was ligated. The apNPY-positive fibers remained strongly stained in the distal end of the ligated nerve, whereas the fibers at the proximal end of the ligated nerve were mostly devoid of staining, suggesting that the apNPY-positive fibers are afferent fibers originating from the gut. Note that the right EN showed a normal staining pattern. $\boldsymbol{F}$, Expanded view of EN from the preparation $\boldsymbol{B 2}$, showing strong staining in the posterior branch (arrow) but not in the anterior branch (arrowhead). 1, 2, 3, Buccal nerve 1, 2, 3. Scale bar: $A, B, 200 \mu \mathrm{m} ; \boldsymbol{C}, \boldsymbol{D}, 50$ $\mu \mathrm{m} ; \boldsymbol{E}, \boldsymbol{F}, 100 \mu \mathrm{m}$.

jneurosci.org as supplemental material). CBI-2, in conjunction with other CBIs, is activated by food stimuli (Rosen et al., 1991; Jing and Weiss, 2005) and thus signals the presence of food. When stimulated, CBI-2 evokes ingestive motor programs. In contrast, the EN communicates information from the esophagus/gut to the buccal CPG (Susswein and Kupfermann, 1975; Schwarz and Susswein, 1986; Kuslansky et al., 1987). Also, EN stimulation elicits egestive programs. Thus, actions of EN stimulation are twofold: activating programs and biasing programs toward egestive ones (Proekt et al., 2004) (supplemental Fig. 2, available at www.jneurosci.org as supplemental material). Here, we sought to determine whether apNPY functions to initiate programs or to modulate programs. We found that 5-8 min perfusion of apNPY in quiescent isolated CNS preparations produced only a few cycles of programs: on average, $0.5 \pm 0.2$ cycles $\left(10^{-6} \mathrm{M} ; n=4\right)$ or 1.5 cycles $\pm 0.3\left(10^{-5} \mathrm{M} ; n=4\right)$ of intermediate or egestive motor programs. In contrast, 5 min stimulation of the EN can evoke, on average, $14.3 \pm 1.5$ cycles of egestive programs $(n=14)$. Thus, apNPY did not play a significant role in initiating motor programs.

To examine the mechanisms that may underlie initiation of motor programs, we examined the effects of EN stimulation on the B63 interneuron, a critical initiator of programs (Hurwitz et al., 1997; Dembrow et al., 2004). B63 was strongly active during EN-elicited egestive programs (Fig. $6 A$, left). Furthermore, each single EN stimulus elicited fast EPSPs in B63, and after repetitive EN stimulation, the EPSPs showed summation and facilitation (Fig. $6 B)$. More importantly, bilateral hyperpolarization of B63 blocked the expression of motor programs as evidenced by the absence of bursting activity in radula closure B8 and weak activity in the I2 nerve (Fig. $6 A$, right) $(n=4)$. Thus, the ability of EN stimulation to initiate programs is likely mediated by its excitatory actions on B63, and these actions are not mediated by apNPY.

\section{Modulatory actions of apNPY on CBI-2-elicited motor programs}

We next sought to examine putative modulatory actions of apNPY on motor programs elicited by the two distinct inputs: CBI-2 and the EN. First, we examined apNPY action on ingestive programs elicited by CBI-2, a command-like interneuron that provides powerful excitatory inputs to buccal CPG interneurons to drive ingestive motor programs (supplemental Fig. 2, available at www.jneurosci.org as supplemental material). To elicit stable motor programs, we evoked single-cycle programs by stimulating $\mathrm{CBI}-2$ at $10 \mathrm{~Hz}$ throughout the protraction phase (I2) with intertrial interval of $1 \mathrm{~min}$. Figure $7 A$ shows a representative example. In normal saline (Fig. 7A1), the program was ingestive, because B8 was primarily active during retraction. However, in $10^{-6} \mathrm{M}$ apNPY, the program first became intermediate (for an example, see Fig. $8 \mathrm{~B}$ ) and then became egestive as $\mathrm{B} 8$ now fired primarily during protraction (Fig. 7A2). 
Subsequently, several minutes after we switched to a higher apNPY concentration $\left(10^{-5} \mathrm{M}\right)$ (Fig. 7A3), B8 activity during protraction became weaker, whereas B8 activity during retraction remained absent. Because ingestive or egestive programs are marked by a substantial activity of closure motoneuron B8 during either retraction or protraction, the data suggest that higher apNPY concentrations may inhibit generation of a functional program. After apNPY washout ( $\sim 40 \mathrm{~min}$ ) (Fig. $7 A 4)$, the program returned to ingestive. The data suggest that apNPY, at lower concentrations, can convert CBI-2elicited ingestive programs to egestive ones, but at higher concentrations, apNPY may have an inhibitory effect.

In Figure $7 B$, we plotted average B8 activity during protraction versus during retraction in three conditions: control, effective concentrations (ECs), and $10^{-5} \mathrm{M}$ (up to $8 \mathrm{~min}$ ), which correspond to Figure $7, A 1, A 2$, and $A 3$, respectively. The EC to complete the switch from ingestion to egestion was $10^{-6} \mathrm{M}$ (Fig. 7A2) for five preparations or after brief application $(\leq 5$ min) of $10^{-5} \mathrm{M}$ apNPY (Fig. $8 C$ ) for the other six preparations, all of which are pooled together as one data point and marked as EC. Group data showed that apNPY had significant effects on B8 activity during retraction (Fig. 7C1) (repeatedmeasures ANOVA; $F_{(3,30)}=97.6 ; p<$ $0.0001 ; n=11)$ and during protraction (Fig. 7C2) $\left(F_{(3,30)}=12.6 ; p<0.0001 ; n=\right.$ 11). Comparisons of the four individual groups with Bonferroni corrections showed that B8 activity during retraction at both EC and $10^{-5} \mathrm{M}$ were significantly decreased compared with control and wash. In contrast, B8 activity during protraction at EC was significantly increased compared with control and wash. B8 activity at $10^{-5} \mathrm{M}$ was significantly decreased compared with that at EC. Furthermore, apNPY treatment shortened protraction duration of CBI-2 programs in a concentration-dependent manner (Fig. $7 D)\left(F_{(3,30)}=\right.$ $25.4 ; p<0.0001 ; n=11)$, consistent with previous findings that protraction duration in $\mathrm{CBI}-2$-elicited egestive programs is shorter than in CBI-2-elicited ingestive programs (Jing et al., 2004).

Because motor neuron activity is controlled by CPG interneurons in the Aplysia feeding circuit (supplemental Fig. 2, available at www.jneurosci.org as supplemental material), we examined how apNPY may have influenced activity levels of two critical CPG neurons that promote specific types of programs: B40 and B20 interneurons (Jing and Weiss, 2001, 2002). Both B40 and B20 are active during protraction, but B40 promotes ingestive patterns by enhancing B8 activity during retraction, whereas B20 promotes egestive patterns by enhancing $\mathrm{B} 8$ activity during protraction. Figure $7 F$ shows that B40 activity decreased in apNPY $\left(F_{(3,9)}=32.7 ; p<0.0001 ; n=4\right)$ and changes in B40 activity paralleled $\mathrm{B} 8$ activity change during retraction (Fig. 7C1). In con- trast, B20 activity increased at EC of apNPY, whereas it decreased at $10^{-5} \mathrm{M}$ (examples in Fig. $7 \mathrm{~A}$ and group data in Fig. $\left.7 F\right)\left(F_{(3,6)}=\right.$ $81.2 ; p<0.0001 ; n=3)$, which also paralleled B8 activity changes during protraction (Fig. 7C2). Furthermore, B4 is a CPG element that is active during the retraction phase and promotes egestive programs by inhibiting B8 (Kabotyanski et al., 1998; Jing and Weiss, 2001; Morgan et al., 2002). Figure 7F shows that compared with control conditions, in the presence of apNPY, B4 activity increased during retraction (Fig. $7 F)\left(F_{(3,18)}=16.1 ; p<0.0001\right.$; $n=7)$, whereas B8 activity changed in the opposite direction (Fig. 7C1). Together with reduced B40 activity, enhanced B4 activity may contribute to reduced B8 activity during retraction. These data indicate that when apNPY converts CBI-2-elicited ingestive programs to egestive programs, the activity of the interneurons that participate in the generation of egestive and ingestive programs undergoes concomitant changes.

Both B18 and apNPY enhance EN-elicited egestive programs Although B18 interneurons have been identified previously (Chiel et al., 1988), their functional role in motor-program 

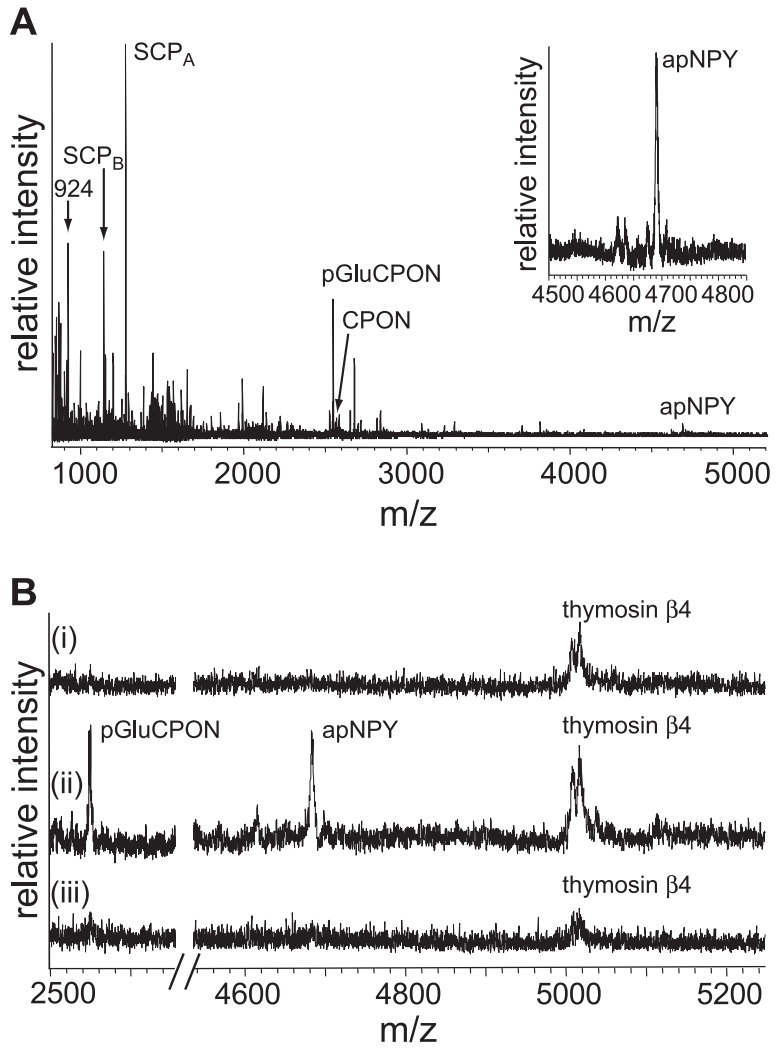

Figure 5. Secretion of apNPY after esophageal nerve stimulation. $\boldsymbol{A}$, The mass spectrum obtained from the EN extract shows the presence of multiple buccal peptides, including $S C P_{A}$ and $\mathrm{SCP}_{\mathrm{B}^{\prime}}$ as well as peptides derived from the Aplysia NPY precursor, including apNPY (see enlarged record in the inset at top right corner), $C P O N$, and pGluCPON. $B$, Mass spectra of buccal releasates showed the presence of apNPY and pGluCPON in bead samples obtained after EN stimulation specifically from the region ipsilateral to stimulation. Compounds such as $\beta 4$ thymosin were observed in all three samples and are considered to be constitutively present and, a priori, independent of EN stimulation. In $\boldsymbol{B}$, releasate mass spectra were obtained from beads collected: (i) before stimulation and from the buccal region ipsilateral to the stimulated EN, (ii) during stimulation and from the buccal region ipsilateral to the stimulated $\mathrm{EN}$, and (iii) during stimulation and from the region contralateral to the stimulated EN. generation has not been examined (for B18 morphology and synaptic connectivity with B20) (Fig. $4 A, B$ ). Here, we determined B18 activity in motor programs elicited by the EN and CBI-2. Interestingly, B18 is strongly active during motor programs elicited by the EN (Fig. 9), consistent with strong excitatory inputs from the EN to B18 (Fig. 4C). Moreover, unlike CPG elements (e.g., B63 and B20) that are typically active during a particular phase (either protraction or retraction) of a program, B18 is active throughout the duration of the EN stimulation and showed a somewhat more pronounced activity during both protraction and retraction and weaker activity between motor programs. In contrast, B18 typically showed weak activity in motor programs elicited by CBI- 2 .

To elucidate a possible role of B18 in motor-program generation, we evoked approximately six cycles of motor programs through continuous stimulation of the EN ( $2 \mathrm{~Hz}$ ) with intertrial intervals of $5 \mathrm{~min}$, either without (Fig. 9A, top) or with (Fig. 9A, bottom) hyperpolarization of bilateral B18. Two of the most apparent effects of B18 hyperpolarization are the decrease of cycle frequency and the increase of program latency. We measured total cycle periods from the second to sixth cycle, excluding the first cycle, because the cycle is typically not fully developed and quite variable as evidenced by weak activity in the B8 neuron and I 2 nerve. The total cycle periods of these five cycles were then converted to average cycle frequency (cycles per minute). For the same reason, we excluded the first cycle and defined the program latency as the time from the onset of EN stimulation to the onset of $\mathrm{I} 2$ activity of the second cycle. Figure $9 A$ illustrates a typical experiment. Statistical analysis of three groups, control ("Ctrl”), B18 hyperpolarization ("B18 hyp"), and "recovery" (Fig. 9C1) shows that cycle frequency of the three groups was significantly different $\left(F_{(2,14)}=35.9 ; p<0.0001 ; n=8\right)$. Furthermore, program latency of the three groups was significantly different (Fig. 9D1) $\left(F_{(2,14)}=33.9 ; p<0.0001 ; n=8\right)$. In contrast, B18 hyperpolarization had no appreciable effects on $\mathrm{B} 8$ activity.

$\mathrm{B} 18$ action on EN-induced egestive programs is intriguing, because B18 is apNPY immunopositive. To examine whether apNPY may mediate, at least in part, the B18 effects, we used the same stimulation paradigm as above and replaced ASW perfusion with ASW containing apNPY. Figure 9B (bottom) shows a typical experiment demonstrating that cycle frequency was increased and program latency was decreased in $10^{-5} \mathrm{M}$ apNPY; group data showed that there were overall differences between Ctrl, $10^{-6} \mathrm{M}$, and $10^{-5} \mathrm{M}$ apNPY, and "wash" groups [cycle frequency: (Fig. 9C2) $F_{(3,12)}=6.6, p<$ 0.01 ; program latency: (Fig. 9D2) $F_{(3,12)}=$ $58, p<0.0001 ; n=5$ ]. Individual comparisons with Bonferroni corrections showed that cycle frequency in $10^{-5} \mathrm{M}$ apNPY differed significantly from the control and wash groups, although the $10^{-6} \mathrm{M}$ apNPY group was not significantly different from the control and wash groups. However, despite the small effect at $10^{-6}$ $\mathrm{M}$ apNPY, during apNPY perfusion of this concentration, hyperpolarization of B18 no longer significantly reduced cycle frequency (cycles per minute) (Fig. 9B, top) (without B18 hyp, $2.69 \pm 0.27$ vs B18 hyp, $2.43 \pm 0.19 ; p>0.05$, paired two-tailed $t$ test; $n=4)$. Similarly, although the pro-
Figure 6. B63 activity is necessary for the initiation of egestive motor programs elicited by the EN stimulation. $A, B 63$ was strongly active during protraction (open bar) of egestive programs elicited by EN stimulation ( $2 \mathrm{~Hz}, 3 \mathrm{~ms}, 9 \mathrm{~V}$ ) (left) and hyperpolarization of bilateral B63 suppressed motor program activity (right). Filled bar, Retraction. Recordings were made in normal saline. B, Left, Single EN stimulation pulse (1.5 ms) elicited fast EPSPs in both ipsilateral and contralateral B63 (c-B63). Right, Repetitive stimulation of EN ( $2 \mathrm{~Hz}$ ) elicited facilitating EPSPs that summated in bilateral B63 s. Recordings were made in highdivalent saline. 


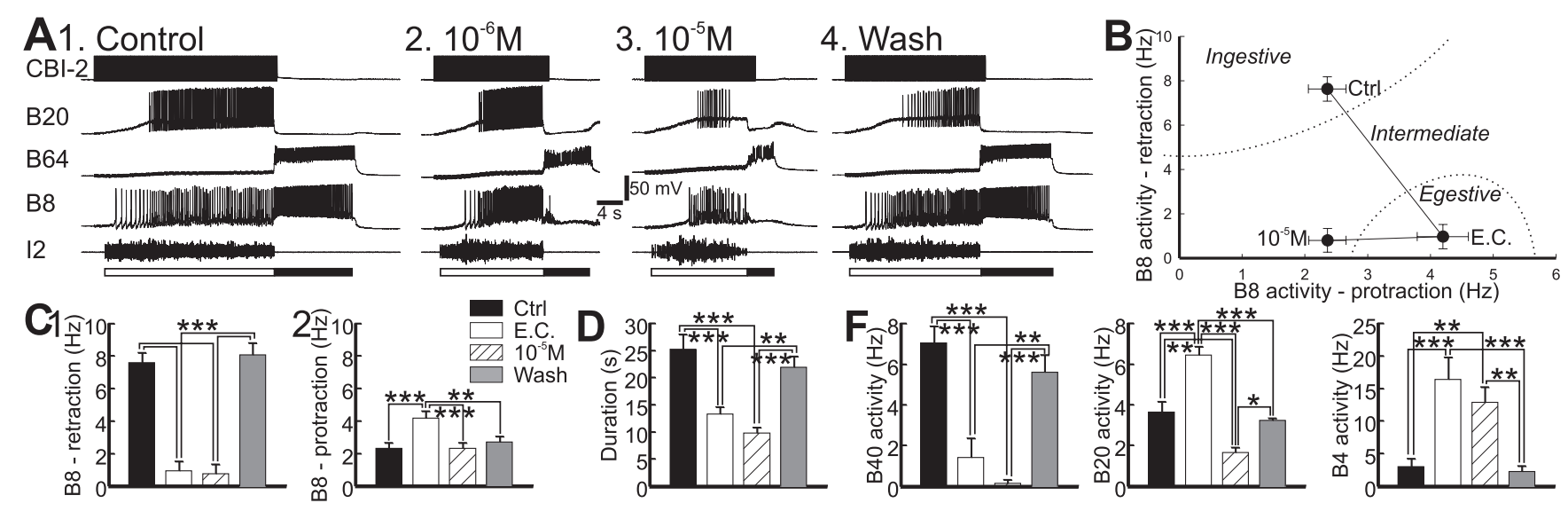

Figure 7. Effects of apNPY on CBI-2-elicited ingestive motor programs. $A$, A representative example. Programs were elicited through stimulation of CBI-2 at $12 \mathrm{~Hz}$ with short current pulses. Protraction (open bar) is monitored by activity in 12 nerve, whereas retraction (filled bar) is monitored by sustained depolarization of the retraction interneuron B64. B20 is a protraction interneuron that promotes firing of the radula closure B8 motoneuron during protraction. The program started as ingestive $(\boldsymbol{A} 1)$ but became egestive in $10^{-6} \mathrm{M}$ apNPY $(\boldsymbol{A} 2)$. In $10^{-5} \mathrm{M}$ apNPY $(\boldsymbol{A 3}), \mathrm{B} 8$ and $\mathrm{B} 20$ activity was suppressed. The program returned to ingestive after $40 \mathrm{~min}$ wash of apNPY (A4). B, Group data. Plot of average B8 activity during protraction versus during retraction of CBI-2-elicited programs $(n=11)$. In control (Ctrl), B8 was mostly active during retraction (ingestive), but at EC ( $10^{-6} \mathrm{M}$ or slightly higher) of apNPY, B8 became mostly active during protraction (egestive). Prolonged exposure ( $>8 \mathrm{~min}$ ) to $10^{-5} \mathrm{M}$ apNPY reduced B8 activity during protraction $\left(10^{-5} \mathrm{M}\right)$. C, Bar graphs of data in $\boldsymbol{B}$ showing statistically significant changes in B8 activity during retraction (C1) and protraction (C2). D, Group data showing apNPY concentration-dependent decrease of protraction duration $(n=11)$. $\boldsymbol{F}$, Group data showing that apNPY altered B40 and B20 activity that matched B8 activity change during retraction and protraction, respectively. apNPY also increased B4 activity during retraction. Post hoc tests with Bonferroni corrections: ${ }^{*} p<0.05$; ${ }^{* *} p<0.01$; ${ }_{* * *}^{*} p 0.001$. The error bar represents SEM.

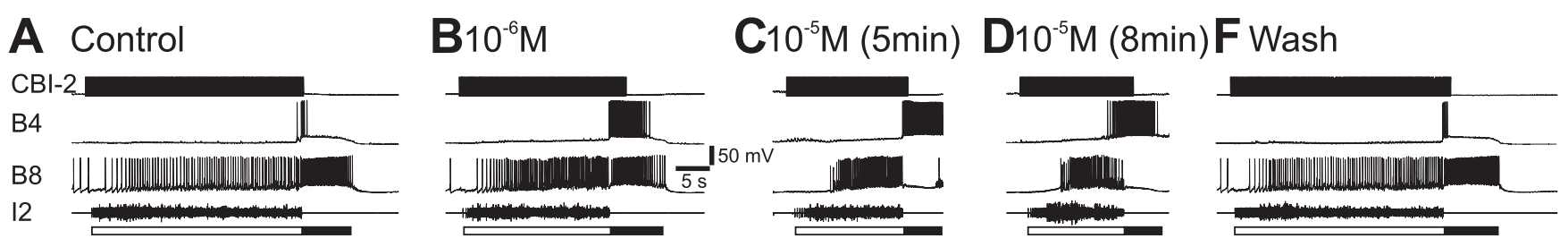

Figure 8. Progression of CBI-2-elicited motor programs during apNPY perfusion illustrates the generation of intermediate programs before the complete conversion to egestive programs. $A$, The motor program elicited by $\mathrm{CBI}-2$ in control conditions was ingestive, because B8 was mostly active during retraction (filled bar). $\boldsymbol{B}$, During the perfusion of $10^{-6} \mathrm{M}$ apNPY, the motor program became intermediate, because B8 was active during both protraction (open bar) and retraction. $C$, After 5 min perfusion of $10^{-5} \mathrm{M}$ apNPY, the motor program became egestive, because B8 was now active only during protraction. $\mathbf{D}, \mathrm{After} 8 \mathrm{~min}$ perfusion of $10^{-5} \mathrm{M}$ apNPY, motor programs became less egestive, because B8 activity during protraction was weaker than in $\boldsymbol{C}$, whereas B8 activity during retraction remained absent. $\boldsymbol{F}$, After an $\sim 35$ min wash, the motor program elicited by CBI-2 became ingestive once again, similar to the control in $\boldsymbol{A}$.

gram latency in the $10^{-6} \mathrm{M}$ apNPY group was not significantly different from the wash group, B18 hyperpolarization during $10^{-6} \mathrm{M}$ apNPY no longer increased and actually slightly decreased, program latency (Fig. 9B, top) (without B18 hyp, $34.29 \pm 2.89 \mathrm{~s}$ vs B18 hyp, $30.72 \pm 3.32 \mathrm{~s} ; n=4)$. In addition, separate analysis of the protraction and retraction durations and interprogram interval suggests that both B18 hyperpolarization and apNPY primarily affected the interprogram interval (Fig. 10). Thus, the data support a causal role of apNPY in mediating the effects of B18.

We conclude that B18 enhances egestive motor programs elicited by the EN by promoting faster cycle frequency and shorter program latency, and the B18 effects are likely mediated by the release of apNPY from B18.

\section{Discussion}

We found that termination of feeding during a shift in the Aplysia internal state from hunger to satiation is effected not simply by inhibition of ingestive responses but by replacement of those responses with nonfunctional (intermediate) and egestive responses. Furthermore, the gradual process leading to a satiety state is implemented by peptidergic reconfiguration of a multifunctional CPG network.
Behavioral and peptidergic aspects of food intake control Peptides are involved in long-term regulation of energy homeostasis of animals (e.g., visceral adiposity signals) (Woods et al., 1998; Badman and Flier, 2005; Morton et al., 2006). However, we focused on short-term control of single meals, which also involves peptidergic mechanisms (Smith, 2000; Badman and Flier, 2005; Cone, 2005; Morton et al., 2006). Among other things, a major peptide target is the feeding CPG, which during a meal receives inhibitory postingestive peptidergic inputs and excitatory orosensory inputs. These excitatory and inhibitory inputs were referred to as direct mechanisms (Smith, 2000) and are thought to play an important role in determining meal size. Here, we studied the Aplysia equivalent of the direct peptidergic inputs.

We concentrated on consummatory feeding responses rather than the more complex appetitive aspects of feeding (Strubbe and Woods, 2004) and endeavored to understand how satiety mediates the rapid modulation of a multifunctional network. We used a hand-feeding technique that allowed us to study primarily the consummatory responses in a manner analogous to handfeeding technique in primates (Yaxley et al., 1985; Rolls et al., 1989) and intraoral infusion in rats (Grill and Norgren, 1978; Kaplan et al., 1990).

When Aplysia were fed to satiation, the fraction of food- 


\section{A Control}

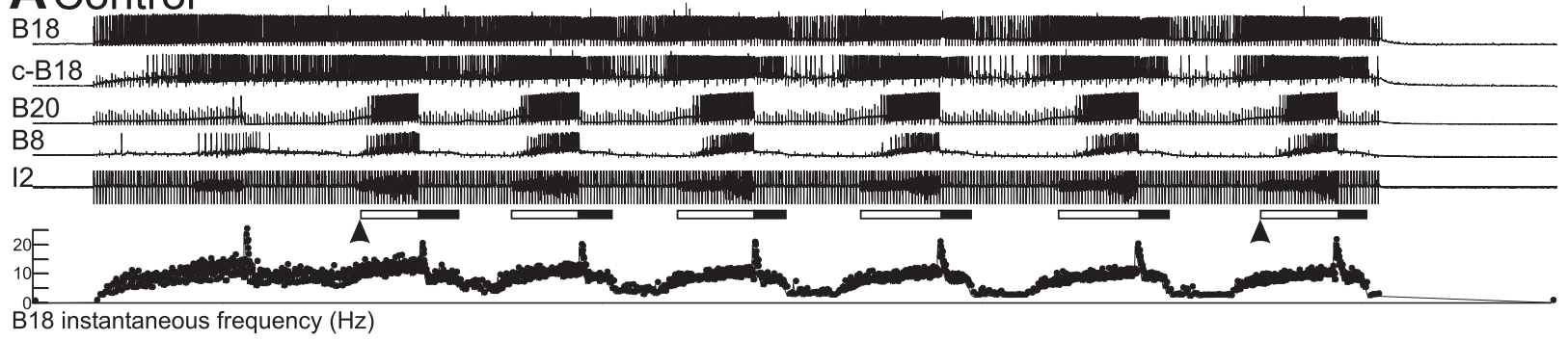

\section{B18 Hyperpolarization}

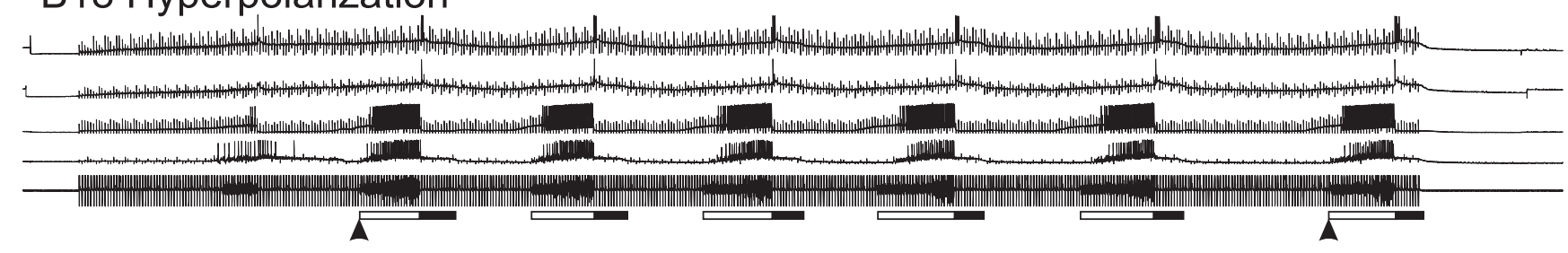

B $10^{-6} \mathrm{M}$ apNPY + B18 Hyperpolarization

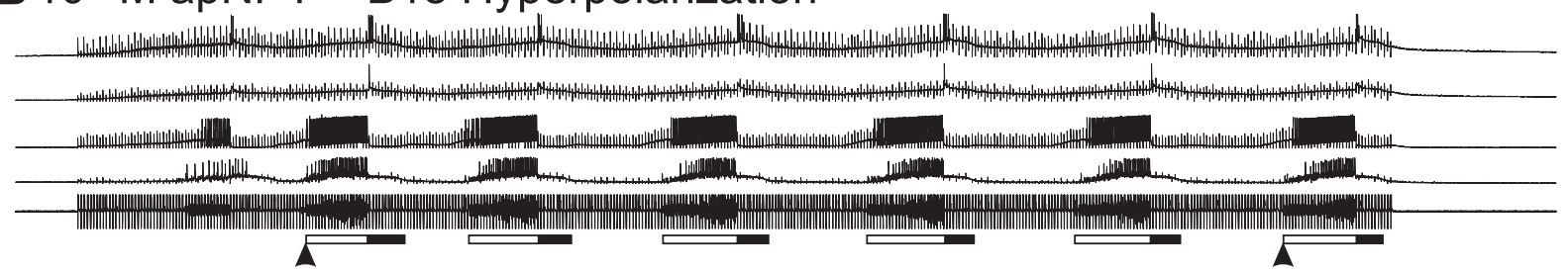

$10^{-5} \mathrm{M}$ apNPY
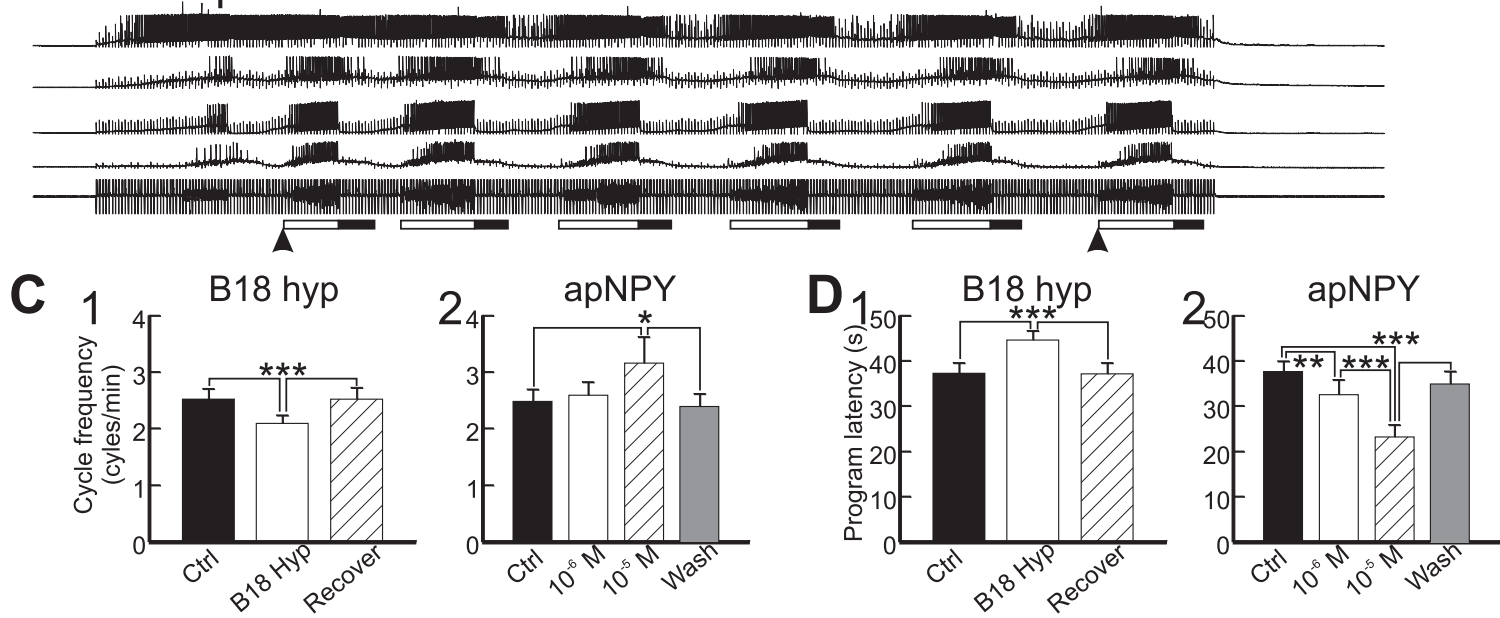

Figure 9. B18 and apNPY enhance EN-elicited egestive programs. $A$, Bilateral B18 s that are ipsilateral and contralateral (c-B18) to the EN were strongly active during EN-elicited egestive programs. Hyperpolarization of bilateral B18 s lengthened the program latency (first arrowhead) and slowed the cycle frequency. $\boldsymbol{B}$, During the perfusion of $10^{-6} \mathrm{M}$ apNPY, B18 hyperpolarization no longer caused longer program latency and slower cycle frequency. In addition, during perfusion of apNPY at $10^{-5} \mathrm{M}$ and in absence of concomitant $\mathrm{B} 18$ hyperpolarization, program latency was shortened further and cycle frequency was sped further. $\boldsymbol{C}, \boldsymbol{D}$, Group data showing the opposite effects of B18 hyperpolarization and apNPY on cycle frequency $(\boldsymbol{C})$ and program latency $(\boldsymbol{D})$. Note that the apNPY data in $\boldsymbol{C}$ and $\boldsymbol{D}$ were from apNPY perfusion experiments without concomitant B18 hyperpolarization. Post hoc tests with Bonferroni corrections: ${ }^{*} p<0.05 ;{ }^{* *} p<0.01 ;{ }^{* * *} p<0.001$. The error bar represents SEM.

elicited ingestive responses progressively decreased, whereas the fractions of intermediate and egestive responses increased until eventually the animals stopped responding to food. The use of chronic electrophysiological recordings enabled us to uncover the emergence of intermediate and egestive responses that might not be directly observable. Furthermore, we observed, as did others (Schwarz et al., 1988), that there was a clear slowing down of feeding, an effect to be expected if ingestive responses were being gradually replaced by intermediate/egestive ones. Thus, processes underlying the transition from hunger to satiation may involve a suppression of ingestive responses and a concomitant facilitation of intermediate/egestive responses.

\section{Role of apNPY in satiation}

Our results indicate that the satiation processes are mediated at least in part by apNPY. In vertebrates, a single ancestral invertebrate-derived NPY-like peptide developed into three major members, NPY, gut peptide PYY, and pancreatic PP, which comprise the vertebrate PP family (Hoyle, 1999). Although hypothalamic NPY strongly promotes feeding in vertebrates (Kalra 

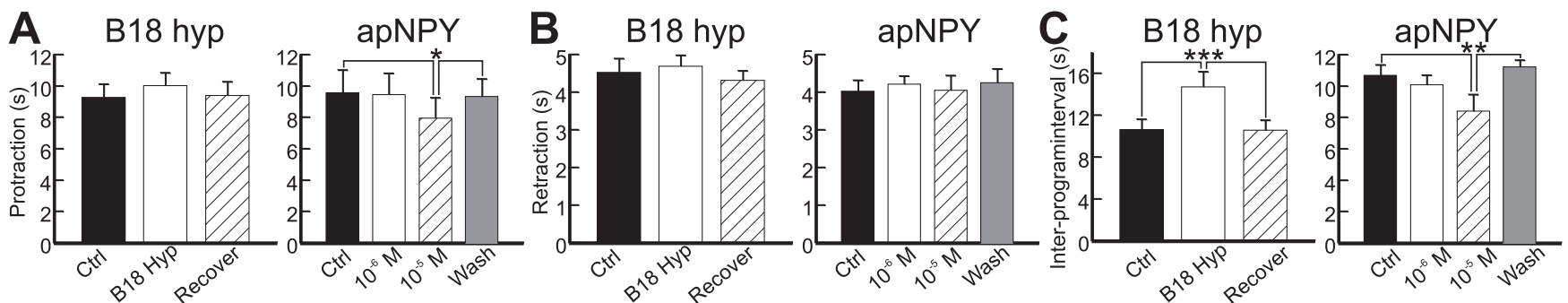

Figure 10. Effects of B18 hyperpolarization and apNPY on parameters of EN-elicited egestive motor programs. As shown in Figure 9 , $A$ and $B$, each cycle of motor programs (between arrowheads) was divided into protraction (open bar), retraction (closed bar), and interprogram interval (time from the end of retraction to the beginning of protraction of the next cycle). Average protraction duration $(\boldsymbol{A})$, retraction duration $(\boldsymbol{B})$, and interprogram interval $(\boldsymbol{C})$ of the five cycles are shown. B18 hyperpolarization (B18 hyp) and apNPY had prominent but opposite effects on the interprogram intervals $(C)\left(B 18\right.$ hyp, $F_{(2,14)}=39.9, p<0.0001, n=8 ;$ apNPY, $\left.F_{(3,12)}=6.67, p<0.01, n=8\right)$, whereas they had no effects on retraction duration $(B)\left(B 18\right.$ hyp, $F_{(2,14)}=2.7, p>0.05, n=$ 8 ; apNPY, $\left.F_{(3,12)}=0.74, p>0.05, n=8\right)$. In contrast, whereas B18 hyperpolarization had no significant effect on protraction duration $\left(F_{(2,14)}=1.86 ; p>0.05 ; n=8\right)$, apNPY (at 10 $\left.{ }^{-5} \mathrm{M}\right)$ had a small but significant effect on protraction duration $(A)\left(F_{(3,12)}=5.8 ; p<0.05 ; n=8\right)$. Post hoc tests with Bonferroni corrections: ${ }^{*} p<0.05 ;{ }^{* *} p<0.01 ;{ }^{* * *} p<0.001$. The error bar represents SEM.
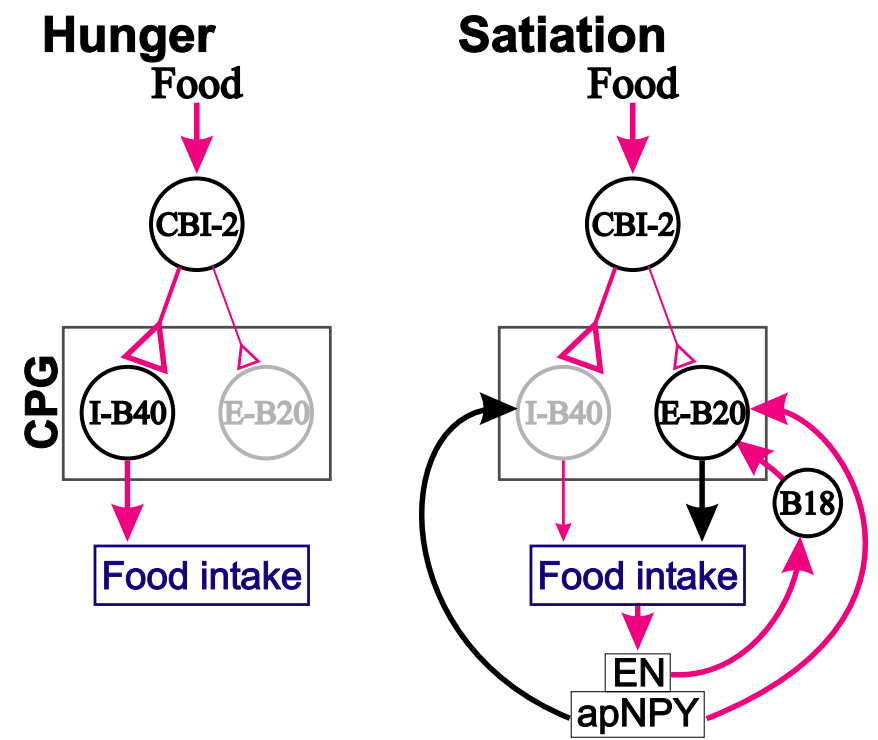

Figure 11. Schematic diagrams showing peptidergic and network mechanisms underlying development of satiety states. Left, When the animals are hungry, food evokes ingestive responses by activating the CPG through $\mathrm{CBI}-2$, which promotes activity of ingestion-promoting B40 interneurons (I-B40). CBI-2 also excites egestion-promoting B20 (E-B20) but less so (connectivity symbol shown in smaller triangle). Right, As a meal progresses and the animal approaches satiation, signals generated from gut activated afferent fibers in the $\mathrm{EN}$, which release apNPY in the buccal ganglion to configure the $\mathrm{CPG}$ to be more permissive to the generation of egestive motor programs, thereby contributing to the establishment of a satiety state and termination of feeding. B18 is also activated by fast excitation from these EN afferent fibers during EN activation, and the apNPY it releases enhances EN-elicited egestive programs by promoting faster cycle frequency and shorter program latency. The black circles around the neuron names denote normal neuronal activity, whereas gray circles denote weak neuronal activity. Open triangles denote synaptic excitation. Red arrows denote activity-promoting pathways, whereas black arrows denote activity-reducing pathways. The large versus small size of arrows denotes strong versus weak effect.

and Kalra, 2004), a form of PYY released from gut inhibits food intake (Batterham et al., 2002). Injection of apNPY in intact Aplysia produced satiation-like effects that manifested in a reduced meal-size and slowing-down of ingestion. It therefore appears that apNPY may be functionally closer to PYY than to other members of this vertebrate peptide family. In contrast, the Drosophila neuropeptide F (NPF), also a homolog of vertebrate PP family, appears to be closely related to the hypothalamic NPY in that NPF essentially promotes feeding, and overexpression of the
NPF receptor seems to promote a more risk-taking feeding strategy (Wu et al., 2005).

Notably, the feeding CPG, like other multifunctional networks (Marder and Calabrese, 1996; Nusbaum and Beenhakker, 2002; Grillner, 2006), can be activated by multiple inputs and can generate multiple outputs. In Aplysia, there are two major input pathways to the CPG, CBI-2 (Rosen et al., 1991; Jing and Weiss, 2005) and the ENs (Schwarz and Susswein, 1986; Kuslansky et al., 1987; Horn et al., 2001), that correspond to the orosensory and postingestive inputs in vertebrates. Direct evidence for EN activation during satiation is currently unavailable, but indirect evidence suggests that ENs are activated, because lesions of ENs (Kuslansky et al., 1987) reduced the ability of bulk stimuli to cause satiation (Susswein and Kupfermann, 1975).

Importantly, nominally identical sensory stimuli, such as food, may evoke different responses depending in part on the current motivational state of the animals (Cabanac, 1971; Kupfermann, 1974). For instance, a short time after a meal, food does not elicit feeding behavior and may even elicit active avoidance (Gillette et al., 2000). In Aplysia, responses elicited by CBI-2 depend in part on the state of the CPG network (Proekt et al., 2004). Thus, in quiescent preparations, CBI-2 stimulation initially elicits intermediate responses and only after repeated CBI-2 stimulation are robust ingestive programs elicited. Perhaps more importantly, after repeated EN stimulation, CBI-2 stimulation elicits egestive rather than ingestive responses.

We localized apNPY in the EN and showed that during EN stimulation, apNPY and other peptides derived from the same prohormone were released within the buccal ganglia where the CPG is located. Furthermore, apNPY mimicked the effects of satiation. As we applied apNPY, CBI-2-elicited ingestive responses were gradually replaced, first by intermediate and then by egestive responses. Notably, this transition was accompanied by decreased activity of B40 and by increased activity of B20. Previous work showed that outputs of the feeding CPG of Aplysia are organized by these two CPG interneurons, B40 and B20, that mediate the competing responses, ingestion and egestion, respectively. Such a neural organization engenders an elegant mechanism where a balance of B40 and B20 activity determines whether the responses are ingestive, intermediate, or egestive (Jing and Weiss, 2001, 2002). Importantly, B40 and B20 do not inhibit each other. Rather, it is the extrinsic signals like apNPY that change the balance of B40 and B20 activity. Therefore, increased release and/or cumulative actions of apNPY during a meal may explain how a gradual transition from a hunger to a satiation state may be 
implemented in the CNS and give rise to the dynamic evolution of feeding responses in intact animals. Thus, the transitions to a satiety state (i.e., cessation of feeding that results from food intake) may be implemented by coordinated peptidergic actions that simultaneously suppress the processes that promote ingestion and enhance those that promote egestion (Fig. 11) (for a more complete circuitry diagram, see supplemental Fig. 2, available at www.jneurosci.org as supplemental material).

A coordinating role of apNPY in satiation is supported by the fact that apNPY acts to enhance EN-elicited programs. apNPYpositive B18 interneuron is strongly active throughout EN stimulation and enhances EN-elicited egestive responses by promoting faster cycle frequency and shorter program latency, which are mimicked and occluded by apNPY. Thus, dual outputs of EN afferents, the direct biasing actions on the CPG and the enhancing action through a feedforward pathway via B18, may ensure that the CPG is biased toward generating robust and frequent egestive programs (Fig. 11).

\section{Conclusions}

Combined with previous studies, our work suggests that the transition from the hunger to satiation states occurs in two stages. The first stage may primarily involve reconfiguration of the feeding CPG. The second stage may primarily entail inhibition of the CPG and possibly other sites (e.g., the appetitive network). According to this hypothesis, in a hungry animal, the CPG would be, by default, configured to generate ingestive behaviors in response to food-elicited activation of CBI-2. During the early stage of a meal, signals generated from the gut would activate afferent fibers in the EN (Kuslansky et al., 1987), leading to a progressively increasing release of apNPY. This would reconfigure the CPG into a mode in which food-elicited activation of CBI-2 increases the fraction of intermediate/egestive responses and reduces the fraction of ingestive responses, thereby reducing food intake (Fig. 11). In the second stage, which we have not studied, feeding is eventually terminated. Previous work suggests that this stage may involve inhibition of the feeding CPG (Staras et al., 2003) as well as higher-order neurons that mediate food-activated input (Davis and Gillette, 1978; Rolls, 2005; de Araujo et al., 2006); however, possible roles of peptides in these inhibitory mechanisms have yet to be determined.

From this perspective, we note that reconfigurative mechanisms may also operate in vertebrates. A transition from ingestion to rejection has been observed in mammals (Grill and Norgren, 1978; Yaxley et al., 1985; Rolls et al., 1989; Cabanac and Lafrance, 1990; Kaplan et al., 1990) when animals were fed to satiation. Furthermore, one prototypical satiety peptide, CCK (Smith, 2000; Badman and Flier, 2005), which appears functionally analogous to apNPY, is released from the gut during a meal, and activates vagal afferents, the activity of which is then relayed to the nucleus of the solitary tract (NTS) and adjacent areas of the brainstem where the afferents act on feeding circuits to terminate a meal. Because NTS and adjacent areas (e.g., reticular formation) comprise afferent paths and circuits for both ingestion and rejection/emesis (Travers et al., 1997; Hornby, 2001), it is possible that during a meal, elements of the CPG that promote responses alternative to ingestion may be enhanced.

In summary, we demonstrated a role of reconfigurative mechanisms of the feeding CPG in the transition from a hunger to a satiety state. More generally, changes in the motivational state of an animal may involve not only simple network inhibition but also reconfiguration of a multifunctional network.

\section{References}

Altschuler SM (2001) Laryngeal and respiratory protective reflexes. Am J Med 111:90S-94S.

Badman MK, Flier JS (2005) The gut and energy balance: visceral allies in the obesity wars. Science 307:1909-1914.

Batterham RL, Cowley MA, Small CJ, Herzog H, Cohen MA, Dakin CL, Wren AM, Brynes AE, Low MJ, Ghatei MA, Cone RD, Bloom SR (2002) Gut hormone PYY(3-36) physiologically inhibits food intake. Nature 418:650-654.

Cabanac M (1971) Physiological role of pleasure. Science 173:1103-1107.

Cabanac M, Lafrance L (1990) Postingestive alliesthesia: the rat tells the same story. Physiol Behav 47:539-543.

Chen Z, Travers JB (2003) Inactivation of amino acid receptors in medullary reticular formation modulates and suppresses ingestion and rejection responses in the awake rat. Am J Physiol Regul Integr Comp Physiol 285:R68-R83.

Chiel HJ, Kupfermann I, Weiss KR (1988) An identified histaminergic neuron can modulate the outputs of buccal-cerebral interneurons in Aplysia via presynaptic inhibition. J Neurosci 8:49-63.

Combes D, Meyrand P, Simmers J (1999) Motor pattern specification by dual descending pathways to a lobster rhythm-generating network. J Neurosci 19:3610-3619.

Cone RD (2005) Anatomy and regulation of the central melanocortin system. Nat Neurosci 8:571-578.

Cropper EC, Brezina V, Vilim FS, Harish O, Price DA, Rosen S, Kupfermann I, Weiss KR (1994) FRF peptides in the ARC neuromuscular system of Aplysia: purification and physiological actions. J Neurophysiol 72:2181-2195.

Davis WJ, Gillette R (1978) Neural correlates of behavioral plasticity in command neurons in Pleurobranchaea. Science 199:801-804.

Davis WJ, Mpitsos GJ, Pinneo JM, Ram JL (1977) Modification of the behavioral hierarchy of Pleurobranchaea. I. Satiation and feeding motivation. J Comp Physiol 117:99-125.

de Araujo IE, Gutierrez R, Oliveira-Maia AJ, Pereira Jr A, Nicolelis MA, Simon SA (2006) Neural ensemble coding of satiety states. Neuron 51:483-494.

Dembrow NC, Jing J, Brezina V, Weiss KR (2004) A specific synaptic pathway activates a conditional plateau potential underlying protraction phase in the Aplysia feeding central pattern generator. J Neurosci 24:5230-5238.

DiNardo LA, Travers JB (1997) Distribution of fos-like immunoreactivity in the medullary reticular formation of the rat after gustatory elicited ingestion and rejection behaviors. J Neurosci 17:3826-3839.

Furukawa Y, Nakamaru K, Wakayama H, Fujisawa Y, Minakata H, Ohta S, Morishita F, Matsushima O, Li L, Romanova E, Sweedler JV, Park JH, Romero A, Cropper EC, Dembrow NC, Jing J, Weiss KR, Vilim FS (2001) The enterins: a novel family of neuropeptides isolated from the enteric nervous system and CNS of Aplysia. J Neurosci 21:8247-8261.

Gillette R, Huang RC, Hatcher N, Moroz LL (2000) Cost-benefit analysis potential in feeding behavior of a predatory snail by integration of hunger, taste, and pain. Proc Natl Acad Sci USA 97:3585-3590.

Grill HJ (2006) Distributed neural control of energy balance: contributions from hindbrain and hypothalamus. Obesity 14:216S-221S.

Grill HJ, Norgren R (1978) Chronically decerebrate rats demonstrate satiation but not bait shyness. Science 201:267-269.

Grillner S (2006) Biological pattern generation: the cellular and computational logic of networks in motion. Neuron 52:751-766.

Groome JR, Clark M, Lent CM (1993) The behavioural state of satiation in the leech is regulated by body distension and mimicked by serotonin depletion. J Exp Biol 182:265-270.

Harris-Warrick RM, Marder E (1991) Modulation of neural networks for behavior. Annu Rev Neurosci 14:39-57.

Hatcher NG, Richmond TA, Rubakhin SS, Sweedler JV (2005) Monitoring activity-dependent peptide release from the CNS using single-bead solidphase extraction and MALDI TOF MS detection. Anal Chem 77:1580-1587.

Horn CC, Kupfermann I (2002) Egestive feeding responses in Aplysia persist after sectioning of the cerebral-buccal connectives: evidence for multiple sites of control of motor programs. Neurosci Lett 323:175-178.

Horn CC, Geizhals CR, Kupfermann I (2001) Further studies of bulk and orosensory decrement in producing satiation of feeding in Aplysia. Brain Res 918:51-59. 
Hornby PJ (2001) Central neurocircuitry associated with emesis. Am J Med 111:106S-112S.

Hoyle CH (1999) Neuropeptide families and their receptors: evolutionary perspectives. Brain Res 848:1-25.

Hurwitz I, Kupfermann I, Susswein AJ (1997) Different roles of neurons B63 and B34 that are active during the protraction phase of buccal motor programs in Aplysia californica. J Neurophysiol 78:1305-1319.

Hurwitz I, Kupfermann I, Weiss KR (2003) Fast synaptic connections from CBIs to pattern-generating neurons in Aplysia: initiation and modification of motor programs. J Neurophysiol 89:2120-2136.

Jing J, Weiss KR (2001) Neural mechanisms of motor program switching in Aplysia. J Neurosci 21:7349-7362.

Jing J, Weiss KR (2002) Interneuronal basis of the generation of related but distinct motor programs in Aplysia: implications for current neuronal models of vertebrate intralimb coordination. J Neurosci 22:6228-6238.

Jing J, Weiss KR (2005) Generation of variants of a motor act in a modular and hierarchical motor network. Curr Biol 15:1712-1721.

Jing J, Vilim FS, Wu JS, Park JH, Weiss KR (2003) Concerted GABAergic actions of Aplysia feeding interneurons in motor program specification. J Neurosci 23:5283-5294.

Jing J, Cropper EC, Hurwitz I, Weiss KR (2004) The construction of movement with behavior-specific and behavior-independent modules. J Neurosci 24:6315-6325.

Kabotyanski EA, Baxter DA, Byrne JH (1998) Identification and characterization of catecholaminergic neuron B65, which initiates and modifies patterned activity in the buccal ganglia of Aplysia. J Neurophysiol 79:605-621.

Kalra SP, Kalra PS (2004) NPY-an endearing journey in search of a neurochemical on/off switch for appetite, sex and reproduction. Peptides 25:465-471.

Kaplan JM, Spector AC, Grill HJ (1990) Ingestion rate as an independent variable in the behavioral analysis of satiation. Am J Physiol 258:R662-R671.

Kristan Jr WB, Calabrese RL, Friesen WO (2005) Neuronal control of leech behavior. Prog Neurobiol 76:279-327.

Kupfermann I (1974) Feeding behavior in Aplysia: a simple system for the study of motivation. Behav Biol 10:1-26.

Kupfermann I, Weiss KR (2001) Motor program selection in simple model systems. Curr Opin Neurobiol 11:673-677.

Kuslansky B, Weiss KR, Kupfermann I (1987) Mechanisms underlying satiation of feeding behavior of the mollusc Aplysia. Behav Neural Biol 48:278-303.

Lieske SP, Thoby-Brisson M, Telgkamp P, Ramirez JM (2000) Reconfiguration of the neural network controlling multiple breathing patterns: eupnea, sighs and gasps. Nat Neurosci 3:600-607.

Lu J, Sherman D, Devor M, Saper CB (2006) A putative flip-flop switch for control of REM sleep. Nature 441:589-594.

Marder E, Calabrese RL (1996) Principles of rhythmic motor pattern generation. Physiol Rev 76:687-717.

Morgan PT, Jing J, Vilim FS, Weiss KR (2002) Interneuronal and peptidergic control of motor pattern switching in Aplysia. J Neurophysiol $87: 49-61$.

Morton DW, Chiel HJ (1993a) In vivo buccal nerve activity that distinguishes ingestion from rejection can be used to predict behavioral transitions in Aplysia. J Comp Physiol 172:17-32.

Morton DW, Chiel HJ (1993b) The timing of activity in motor neurons that produce radula movements distinguishes ingestion from rejection in Aplysia. J Comp Physiol 173:519-536.

Morton GJ, Cummings DE, Baskin DG, Barsh GS, Schwartz MW (2006) Central nervous system control of food intake and body weight. Nature 443:289-295.

Nusbaum MP, Beenhakker MP (2002) A small-systems approach to motor pattern generation. Nature 417:343-350.

Proekt A, Brezina V, Weiss KR (2004) Dynamical basis of intentions and expectations in a simple neuronal network. Proc Natl Acad Sci USA 101:9447-9452.

Rajpara SM, Garcia PD, Roberts R, Eliassen JC, Owens DF, Maltby D, Myers RM, Mayeri E (1992) Identification and molecular cloning of a neuropeptide Y homolog that produces prolonged inhibition in Aplysia neurons. Neuron 9:505-513.

Reingold SC, Gelperin A (1980) Feeding motor programme in Limax. II. Modulation by sensory inputs in intact animals and isolated central nervous systems. J Exp Biol 85:1-19.

Rolls ET (2005) Taste, olfactory, and food texture processing in the brain, and the control of food intake. Physiol Behav 85:45-56.

Rolls ET, Sienkiewicz ZJ, Yaxley S (1989) Hunger modulates the responses to gustatory stimuli of single neurons in the caudolateral orbitofrontal cortex of the macaque monkey. Eur J Neurosci 1:53-60.

Romanova EV, Jakubowski JA, Vilim FS, Weiss KR, Sweedler JV (2006) Analysis of neuropeptide Y prohormone processing in Aplysia neurons by mass spectrometry. Soc Neurosci Abstr 32:129.24.

Rosen SC, Teyke T, Miller MW, Weiss KR, Kupfermann I (1991) Identification and characterization of cerebral-to-buccal interneurons implicated in the control of motor programs associated with feeding in Aplysia. J Neurosci 11:3630-3655.

Schwartz MW, Woods SC, Porte Jr D, Seeley RJ, Baskin DG (2000) Central nervous system control of food intake. Nature 404:661-671.

Schwarz M, Susswein AJ (1986) Identification of the neural pathway for reinforcement of feeding when Aplysia learn that food is inedible. J Neurosci 6:1528-1536.

Schwarz M, Markovich S, Susswein AJ (1988) Parametric features of inhibition of feeding in Aplysia by associative learning, satiation, and sustained lip stimulation. Behav Neurosci 102:124-133.

Smith GP (2000) The controls of eating: a shift from nutritional homeostasis to behavioral neuroscience. Nutrition 16:814-820.

Staras K, Kemenes I, Benjamin PR, Kemenes G (2003) Loss of self-inhibition is a cellular mechanism for episodic rhythmic behavior. Curr Biol 13:116-124.

Strand FL (1999) Neuropeptides: regulators of physiological processes. Cambridge, MA: MIT.

Strubbe JH, Woods SC (2004) The timing of meals. Psychol Rev 111:128-141.

Susswein AJ, Kupfermann I (1975) Bulk as a stimulus for satiation in Aplysia. Behav Biol 13:203-209.

Sweedler JV, Li L, Rubakhin SS, Alexeeva V, Dembrow NC, Dowling O, Jing J, Weiss KR, Vilim FS (2002) Identification and characterization of the feeding circuit-activating peptides, a novel neuropeptide family of Aplysia. J Neurosci 22:7797-7808.

Travers JB, Dinardo LA, Karimnamazi H (1997) Motor and premotor mechanisms of licking. Neurosci Biobehav Rev 21:631-647.

Vilim FS, Alexeeva V, Moroz LL, Li L, Moroz TP, Sweedler JV, Weiss KR (2001) Cloning, expression and processing of the CP2 neuropeptide precursor of Aplysia. Peptides 22:2027-2038.

Woods SC, Seeley RJ, Porte Jr D, Schwartz MW (1998) Signals that regulate food intake and energy homeostasis. Science 280:1378-1383.

Wu Q, Zhao Z, Shen P (2005) Regulation of aversion to noxious food by Drosophila neuropeptide Y- and insulin-like systems. Nat Neurosci 8:1350-1355.

Xin Y, Hurwitz I, Perrins R, Evans CG, Alexeeva V, Weiss KR, Kupfermann I (1999) Actions of a pair of identified cerebral-buccal interneurons (CBI8/9) in Aplysia that contain the peptide myomodulin. J Neurophysiol 81:507-520.

Yaxley S, Rolls ET, Sienkiewicz ZJ, Scott TR (1985) Satiety does not affect gustatory activity in the nucleus of the solitary tract of the alert monkey. Brain Res 347:85-93.

Zhurov Y, Proekt A, Weiss KR, Brezina V (2005) Changes of internal state are expressed in coherent shifts of neuromuscular activity in Aplysia feeding behavior. J Neurosci 25:1268-1280. 